\title{
Lies, Damn Lies, and Pre-Election Polling
}

\author{
Elias Walsh \\ University of Michigan, Ann Arbor, \\ fgelias@umich.edu, \\ Sarah Dolfin \\ Mathematica Research Inc., \\ SDolfin@mathematica-mpr.com, \\ and John DiNardo, \\ University of Michigan, Ann Arbor and NBER \\ jdinardo@umich.edu
}

\author{
Corresponding Author: John DiNardo \\ Session Title: Polls, Votes, and Elections \\ Session Chair: JOHN DINARDO, University of Michigan \\ Discussants: MIREILLE JACOBSON, University of California- \\ Irvine \\ HEATHER ROYER, University of California, Santa Barbara
}

\section{Web Appendices:}

1. Data appendix

2. Ten (10) web appendix tables.

3. Eleven (11) web appendix figures.

4. Five page discussion of intentions problem.

5. Poll questions

January 30, 2009 


\section{Web-Appendices}

1) Appendix 1. Data Appendix.

2) Appendix 2. Ten (10) Web Tables.

3) Appendix 3. Eleven (11) Web Figures.

4) Appendix 4. Short Discussion of Intentions.

5) Appendix 5. Poll Questions. 


\title{
Web Appendix: Data
}

\author{
Elias Walsh, Sarah Dolfin and John DiNardo
}

January 30, 2009

\section{Election Poll Data}

We include all general election polls including at minimum both of the major party candidates and completed after June 1 of the election year. We identify and drop polls reported multiple times. When a single poll reports responses to the question phrased to allow third party candidates and another question phrased to force a choice between the Democratic and Republican candidates we use only the poll that allows the respondent more options. When a poll reports the results of the full sample in addition to some number of subsamples we use only the sample that limits respondents to "likely voters." Finally, we drop 39 polls with no reported sample size.

\section{Election Results Data}

We obtained official 1996, 2000 and 2004 presidential election results from the Federal Election Commission website: accessed http://www.fec.gov/pubrec/fe1996/presge.htm on February 11, 2008 accessed http://www.fec.gov/pubrec/2000presgeresults.htm on February 11, 2008 accessed http://www . fec.gov/pubrec/fe2004/federalelections2004.pdf on February 11, 2008 According to the FEC these results are "the official, certified federal election results obtained from each state's election office and other official sources." http://www.fec.gov/pubrec/electionresults.shtml. Official results of the 2008 presidential election were not yet available at the time of this writing. For this election we obtain results from the most up-to-date tallies from media websites or from the state Secretary of State office when available. These results are conveniently available with sources from Wikipedia.com (accessed from http://en.wikipedia.org/wiki/2008_presidential_election on November 19, 2008).

\section{TESS Poll Data}

The data and documentation for our survey, conducted by TESS and Knowledge Networks is available at http://www.experimentcentral.org/data/data.php?pid=298. The first wave was conducted between October 19th and October 24th, 2004. The second wave was conducted between October 26th and November 1st, 2004. We drop four observations from the Manski group with no response for probability of voting (three of these also have missing poll results). We also drop a combined 58 observations from both groups with missing poll results. The survey completion rate is $68 \%$ for the first wave and $71 \%$ for the second wave. 


\title{
Web Appendix: Tables
}

\author{
Elias Walsh, Sarah Dolfin and John DiNardo
}

January 30, 2009

\begin{abstract}
Web Appendix Table 1: November "Trial Heats" for 2000 U.S. Presidential Election Of these 43 "last minute" national horse race polls from the 2000 U.S. Presidential Election only 3 of the 42 polls predicted either a "tie" or Gore ahead in the national race, despite the fact that the actual vote was a virtual "tie" (with Gore actually winning the popular vote). Consultation of the tables for the binomial distribution reveals that the probability of 42 or more "Bush" predictions out of the 45 displayed above is less than $5 \times 10^{-7}$ percent. In making this calculation we use the assumption that Gore (the Democratic candidate) and Bush (the Republican candidate) received exactly the same number of votes, and the polls were independent samples.
\end{abstract}

Web Appendix Table 2: Descriptive Statistics of Pre-Election Poll Sample, 2000-2008 The implied sample size is calculated from the reported margin of error and a mean of 0.50. Similarly, the implied margin of error is calculated from the reported sample size and mean of 0.50 . The differences between the reported and implied values can be attributed to rounding error in most (but not all) cases. The sample includes all available statewide pre-election polls completed on or after the first day of June in the election year. We drop 39 polls with missing sample size from all analyses. See text for a further discussion of the sample inclusion criteria. Over a third of all polls in our sample are conducted within two weeks of election day, and approximately $85 \%$ of polls are reported as polls of "likely voters" (as opposed to registered voters, adults, or no qualification at all). The intensity of polling by state tends to increase across the three election years, with a median (mean) of 9 (13.5) polls per state in 2008 and a median (mean) of 5 (10.1) polls per state in 2000.

Web Appendix Table 3: Total Percentage Reported in Polls

The poll totals in this table include all reported categories including undecided and other candidate respondents. The sum of the predicted shares in many polls do not add up to exactly 100 percentage points. Since nearly all polls report figures rounded to two digits, many of these sums can be explained by rounding error. We do observe a small fraction of polls that sum to an amount below that which can be explained by rounding error, although over $95 \%$ of the polls in our sample do add up to 99 percentage points or higher. In these cases, as in the case of rounding error, we handle the problem symmetrically to the undecided problem and use the share of the total reported poll as the prediction (see text for details).

\section{Polls}

Web Appendix Table 4: Descriptive Statistics for Undecideds and Other Candidates in "Conditional" shares are conditional on being having any undecided or ambiguous respondents (or third party, other or none in bottom panel). "Ambiguous" shares include categories that are lumped together, such as "Other/Undecided" as well as shares left unaccounted. The vote shares are the unweighted means across polls. Only about $1 \%$ of polls have no undecided or ambiguous respondents. In polls with undecided or ambiguous respondents these respondents account for approximately $7 \%$ of the total, most of whom are classified as undecided. The fraction of polls with third party candidates varies with the election year. In the 2000 election $3.7 \%$ of the electorate voted for a third party candidate, while only about $1 \%$ did so in 2004 or 2008. As might be expected, the 2000 polls included third party candidates (or other/none) over $90 \%$ of the time, while 2008 polls included these only about $70 \%$ of the time. The composition of the third party candidate components varies by election year.

Web Appendix Table 5: Pre-Election Polls

"Adj" means treating undecided respondents as strongly ignorable. The standardized prediction errors are calculated using the equation in the text. Under the null that the poll results are i.i.d. draws from the true distribution, the mean of the standardized prediction error is 0 and the variance is 1 . Prediction errors and shares are in units of percentage points. See text for a discussion of this table.

Web Appendix Table 6: Pre-Election Polls, by Year

"Adj" means treating undecided respondents as strongly ignorable. The standardized prediction errors are calculated using the equation in the text. Under the null that the poll results are i.i.d. draws from the true distribution, the mean of the standardized prediction error is 0 and the variance is 1 . Prediction errors and shares are in units of percentage points. The pattern of over-dispersion and bias is consistent across election years. The polls in 2004 are slightly less disperse and display the least bias of the three years. As noted in the text, the 2004 race was, to a large extent, a "replay" of the 2000 election, possibly making the 2004 election easier to predict. Indeed, use of the 2000 election result as a prediction would have correctly 
guessed the winner $94 \%$ of the time: the polls we analyzed guessed the victor less than $74 \%$ of the time. The fact that most polls are conducted for "hard to predict races" only partly explains this fact, since even accounting for where polls are conducted, the 2000 election result will correctly guess the winner $83 \%$ of the time. In the 2000 and 2008 races the polls outperform this crude benchmark, but not by a large margin.

Web Appendix Table 7: Error in Pre-Election Polls, By Inclusion of Third Party Candidates All columns treat undecided respondents as strongly ignorable. Under the null that the poll results are i.i.d. draws from the true distribution, the mean of the standardized prediction error is 0 and the variance is 1 . Prediction errors and shares are in units of percentage points. Third party candidates received $1.3 \%$ of the popular vote in $2008,1.0 \%$ in 2004 and $3.7 \%$ in 2000 . In the 2000 elections polls that included any third party candidate provided forecasts with more bias for the Democratic candidate, less bias for the Republican candidate, and much less disperse forecasts for both. However, in 2004 we see precisely the opposite pattern.

Web Appendix Table 8: The Relation Between Forecast Errors and Prior Information

Each column is an OLS regression clustered by state. The dependent variable is the adjusted poll result, treating undecided respondents as strongly ignorable. See text for a discussion of the Democratic candidate results. The results are qualitatively similar for the Republican candidate results with somewhat less weight placed on the prior than for the Democratic candidate, though this difference is not statistically significant.

Web Appendix Table 9: Descriptive Statistics of Manski Poll

See text for a discussion of the TESS poll. The table demonstrates that the means of observed individual characteristics do not differ significantly within wave across the treatment and control groups with a p-value of 0.34 in wave 1 and 0.90 in wave 2. Approximately $85 \%$ of the control group sample responded that they intended to vote in the election. This fraction is statistically indistinguishable from the mean of the reported probability of voting in the Manski group sample. Over $75 \%$ of the Manski sample reported that they were virtually certain of going to the polls. A similar fraction also expressed certainty about their choice of candidate. With so few respondents expressing uncertainty about their voting behavior one might be surprised to see important differences in the estimated preferences of the experimental groups.

Web Appendix Table 10: Probabilistic vs. Usual Style Questions Results pool both survey waves, employing DFL weights to account for differences in observed sample demographics between waves. In addition, we employ the survey weights provided by TESS designed to match the demographics of the surveyed sample to the U.S. Census and the Knowledge Networks Panel. The likely voter weights use the reported probability of voting (for the Manski group only) to adjust results. The missing data weights use DFL weights to account for 58 dropped observations with missing poll results on observed dimensions of demographics. Actual national 2004 election results were Bush $50.733 \%$, Kerry $48.270 \%$, and Other $0.996 \%$. See text for discussion of pooled results. The results tabulated separately by wave do not demonstrate any significant differences between the Manski and the control group respondents. 
Web Appendix Table 1: November "Trial Heats" for 2000 U.S. Presidential Election

\begin{tabular}{|c|c|c|c|c|c|c|}
\hline$\overline{\text { Date }}$ & Size & & Gore & $\overline{\text { Bush }}$ & 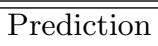 & Polling Agency \\
\hline $11 / 3-5$ & 1801 & & 45.9 & 49.0 & False & ABC News \\
\hline $11 / 2-4$ & 1741 & & 45.9 & 50.0 & False & Poll \\
\hline $11 / 1-3$ & 1495 & & 46.9 & 50.0 & False & \\
\hline $10 / 31-11 / 2$ & 1280 & & 45.9 & 49.0 & False & \\
\hline $10 / 30-11 / 1$ & 1032 & & 46.4 & 50.5 & False & \\
\hline $11 / 5-6$ & 2350 & & 46.0 & 48.0 & False & Gallup/CNN \\
\hline $11 / 5-6$ & 2350 & $\dagger^{a}$ & 46.4 & 48.5 & False & USA Today \\
\hline $11 / 4-5$ & 2386 & & 46.4 & 48.5 & False & Poll \\
\hline $11 / 2-4$ & 2733 & & 44.8 & 50.0 & False & \\
\hline $11 / 1-3$ & 2222 & & 45.3 & 49.5 & False & \\
\hline $10 / 31-11 / 2$ & 2128 & & 44.2 & 50.5 & False & \\
\hline $10 / 30-11 / 1$ & 2123 & & 45.3 & 49.5 & False & \\
\hline $11 / 1-2$ & 623 & & 45.8 & 51.0 & False & Marist College \\
\hline $11 / 3-5$ & 1026 & & 45.8 & 49.0 & False & NBC News/Wall \\
\hline $11 / 2-3$ & 751 & & 45.4 & 48.5 & False & Street Journal \\
\hline $10 / 31-11 / 2$ & 808 & & 46.2 & 48.4 & False & Newsweek Poll \\
\hline $11 / 2-5$ & 1301 & & 47.0 & 49.0 & False & Pew Research Center \\
\hline $11 / 2-5$ & 1301 & $\dagger$ & 46.7 & 48.9 & False & for the People \& the \\
\hline $11 / 1-4$ & 1307 & & 46.2 & 49.5 & False & Press Survey \\
\hline $11 / 4-6$ & 1091 & & 47.9 & 46.8 & True & CBS News Poll \\
\hline $11 / 2-5$ & 1273 & & 44.7 & 48.9 & False & \\
\hline $11 / 1-3$ & 825 & & 46.3 & 48.4 & False & \\
\hline $11 / 1-4$ & 1158 & & 44.2 & 49.5 & False & CBS News/New York Times Poll \\
\hline $11 / 1-2$ & 1000 & & 47.8 & 47.8 & False & Fox News/Opinion Dynamics Poll \\
\hline $11 / 3-5$ & 1348 & & 47.0 & 47.0 & True & The Harris Poll \\
\hline $11 / 1-5$ & $\S^{b}$ & & 44.4 & 46.5 & False & ICR \\
\hline $11 / 5-6$ & 1000 & & 45.0 & 50.0 & False & Tarrance Group-d-/ \\
\hline $11 / 5-6$ & 1000 & $\dagger$ & 45.6 & 51.1 & False & Lake Snell Perry \& Assoc.-R- \\
\hline $11 / 1-2,5$ & 1000 & & 41.6 & 51.7 & False & Voter.com/ \\
\hline $10 / 30-11 / 2$ & 1000 & & 41.6 & 51.7 & False & Battleground Survey \\
\hline $10 / 29-31,11 / 1$ & 1000 & & 43.3 & 51.1 & False & \\
\hline $11 / 4-6$ & 1292 & & 46.0 & 47.9 & False & Christian Science Monitor/ \\
\hline $11 / 3-5$ & 989 & & 44.7 & 51.1 & False & Investor's Business Daily/ \\
\hline $11 / 2-4$ & 718 & & 42.4 & 52.2 & False & TIPP Poll \\
\hline $11 / 1-3$ & 838 & & 41.4 & 48.5 & False & \\
\hline $10 / 31-11 / 2$ & 1070 & & 42.4 & 47.5 & False & \\
\hline $10 / 30-11 / 1$ & 1186 & & 45.3 & 50.5 & False & \\
\hline $11 / 3-5$ & 1253 & & 45.2 & 48.4 & False & Hotline Bullseye Poll \\
\hline $10 / 31-11 / 2$ & 1000 & & 43.0 & 50.5 & False & \\
\hline $11 / 4-6$ & 1200 & $\ddagger^{c}$ & 48.0 & 46.0 & True & Reuters/MSNBC \\
\hline $11 / 3-5$ & 1200 & $\ddagger$ & 46.0 & 47.0 & False & Tracking Poll \\
\hline $11 / 2-4$ & 1200 & $\ddagger$ & 44.4 & 46.5 & False & \\
\hline $11 / 1-3$ & 1200 & $\ddagger$ & 44.2 & 48.4 & False & \\
\hline $10 / 30-11 / 2$ & 1200 & $\ddagger$ & 45.2 & 48.4 & False & \\
\hline $10 / 29-11 / 1$ & 1200 & $\ddagger$ & 42.4 & 45.5 & False & \\
\hline
\end{tabular}

${ }^{a}$ This poll is a duplicate of the one immediately above but applies allocation algorithm as if true allocated had not been reported. In principle, they should differ only because of rounding error.

${ }^{b}$ No sample size reported.

${ }^{c}$ Only "approximate" sample size reported

${ }^{c}$ Source pollingreport.com. 
Web Appendix Table 2: Descriptive Statistics of Pre-Election Poll Sample, 2000-2008

\begin{tabular}{|c|c|c|c|c|}
\hline & All Polls & 2000 Polls & 2004 Polls & 2008 Polls \\
\hline Days before election & $\begin{array}{c}40.23 \\
\{39.01\}\end{array}$ & $\begin{array}{c}38.02 \\
\{41.71\}\end{array}$ & $\begin{array}{c}41.48 \\
\{40.08\}\end{array}$ & $\begin{array}{c}40.47 \\
\{35.77\}\end{array}$ \\
\hline$<$ two weeks before election & 0.38 & 0.48 & 0.37 & 0.32 \\
\hline Poll of "likely voters" & 0.84 & 0.85 & 0.83 & 0.83 \\
\hline Reported sample size & $\begin{array}{c}697.07 \\
\{280.32\}\end{array}$ & $\begin{array}{c}626.82 \\
\{213.26\}\end{array}$ & $\begin{array}{c}733.20 \\
\{276.62\}\end{array}$ & $\begin{array}{c}708.72 \\
\{314.96\}\end{array}$ \\
\hline Reported margin of error & $\begin{array}{c}3.86 \\
\{0.61\}\end{array}$ & $\begin{array}{c}4.07 \\
\{0.57\}\end{array}$ & $\begin{array}{c}3.73 \\
\{0.56\}\end{array}$ & $\begin{array}{c}3.85 \\
\{0.64\}\end{array}$ \\
\hline Implied sample size & $\begin{array}{c}703.76 \\
\{281.71\}\end{array}$ & $\begin{array}{c}620.31 \\
\{226.98\}\end{array}$ & $\begin{array}{c}743.96 \\
\{266.46\}\end{array}$ & $\begin{array}{c}715.54 \\
\{316.47\}\end{array}$ \\
\hline Implied margin of error & $\begin{array}{c}3.88 \\
\{0.65\}\end{array}$ & $\begin{array}{c}4.04 \\
\{0.56\}\end{array}$ & $\begin{array}{c}3.74 \\
\{0.50\}\end{array}$ & $\begin{array}{c}3.90 \\
\{0.81\}\end{array}$ \\
\hline Number of polls & 1857 & 475 & 705 & 677 \\
\hline Number of races & 143 & 47 & 46 & 50 \\
\hline Mean polls per race & 12.99 & 10.11 & 15.33 & 13.54 \\
\hline Median polls per race & 7 & 5 & 7.5 & 9 \\
\hline Minimum polls per race & 1 & 1 & 1 & 1 \\
\hline Maximum polls per race & 80 & 37 & 64 & 80 \\
\hline
\end{tabular}

The implied sample size is calculated from the reported margin of error and a mean of 0.50. Similarly, the implied margin of error is calculated from the reported sample size and mean of 0.50. The differences between the reported and implied values can be attributed to rounding error in most (but not all) cases. The sample includes all available state-level pre-election polls completed on or after the first day of June in the election year. We drop 39 polls with missing sample size from all analyses. See text for a further discussion of the sample inclusion criteria. The source for all polls is pollingreport.com. Standard deviations in braces.

Web Appendix Table 3: Total Percentage Reported in Polls

\begin{tabular}{|c|c|c|c|c|}
\hline & All Polls & 2000 Polls & 2004 Polls & 2008 Polls \\
\hline Mean & 99.82 & 99.85 & 100.02 & 99.58 \\
\hline Standard Deviation & 1.39 & 0.81 & 0.58 & 2.11 \\
\hline Minimum & 81 & 89 & 92 & 81 \\
\hline 5th percentile & 99 & 99 & 99 & 98 \\
\hline 10th percentile & 99 & 99 & 100 & 99 \\
\hline 25th percentile & 100 & 100 & 100 & 100 \\
\hline 90th percentile & 101 & 100 & 101 & 101 \\
\hline 95th percentile & 101 & 101 & 101 & 101 \\
\hline Maximum & 102 & 102 & 102 & 102 \\
\hline Number of polls & 1857 & 475 & 705 & 677 \\
\hline
\end{tabular}

Poll totals include all reported categories including undecided and other candidate respondents. 
Web Appendix Table 4: Descriptive Statistics for Undecideds and Other Candidates in Polls

\begin{tabular}{|c|c|c|c|c|}
\hline & All Polls & 2000 Polls & 2004 Polls & 2008 Polls \\
\hline $\begin{array}{l}\text { Fraction of polls with any } \\
\text { undecided or ambiguous }\end{array}$ & 0.989 & 0.981 & 0.996 & 0.987 \\
\hline Share of poll (conditional) & 0.074 & 0.092 & 0.064 & 0.073 \\
\hline \multicolumn{5}{|l|}{ Vote shares (conditional) } \\
\hline Undecided & 0.057 & 0.069 & 0.053 & 0.054 \\
\hline Ambiguous & 0.014 & 0.021 & 0.010 & 0.013 \\
\hline Unaccounted & 0.003 & 0.003 & 0.001 & 0.005 \\
\hline $\begin{array}{l}\text { Fraction of polls with any } \\
\text { third party, other or none }\end{array}$ & 0.793 & 0.914 & 0.804 & 0.697 \\
\hline Share of poll (conditional) & 0.033 & 0.053 & 0.023 & 0.028 \\
\hline \multicolumn{5}{|l|}{ Vote shares (conditional) } \\
\hline Green & 0.012 & 0.039 & 0.000 & 0.000 \\
\hline Independent & 0.008 & 0.000 & 0.015 & 0.006 \\
\hline Libertarian & 0.002 & 0.001 & 0.000 & 0.004 \\
\hline Reform & 0.003 & 0.010 & 0.000 & 0.000 \\
\hline Constitution & 0.000 & 0.000 & 0.000 & 0.000 \\
\hline Other & 0.009 & 0.004 & 0.007 & 0.016 \\
\hline None & 0.001 & 0.000 & 0.000 & 0.001 \\
\hline
\end{tabular}

"Conditional" shares are conditional on having any undecided or ambiguous respondents (or third party, other or none in bottom panel). "Ambiguous" shares include categories that are lumped together, such as "Other/Undecided" as well as shares left unaccounted. Vote shares are the unweighted means across polls. 
Web Appendix Table 5: Pre-Election Polls

\begin{tabular}{|c|c|c|c|c|c|c|}
\hline \multirow[b]{3}{*}{ Republican share } & \multicolumn{2}{|c|}{ All Polls } & \multicolumn{2}{|c|}{ "Likely Vote } & \multicolumn{2}{|c|}{$\begin{array}{c}<2 \text { Weeks before } \\
\text { Election } \\
\mathrm{N}=704\end{array}$} \\
\hline & Raw & Adj & Raw & Adj & Raw & Adj \\
\hline & $\begin{array}{l}48.17 \\
\{6.12\}\end{array}$ & & $\begin{array}{c}48.21 \\
\{5.90\}\end{array}$ & & $\begin{array}{r}48.31 \\
\{5.36\}\end{array}$ & \\
\hline Democratic share & $\begin{array}{r}49.99 \\
\{5.93\}\end{array}$ & & $\begin{array}{r}49.98 \\
\{5.66\}\end{array}$ & & $\begin{array}{r}49.75 \\
\{5.15\}\end{array}$ & \\
\hline Predicted Republican & $\begin{array}{r}44.70 \\
\{5.99\}\end{array}$ & $\begin{array}{l}48.20 \\
\{6.31\}\end{array}$ & $\begin{array}{r}45.03 \\
\{5.71\}\end{array}$ & $\begin{array}{l}48.31 \\
\{6.00\}\end{array}$ & $\begin{array}{l}45.14 \\
\{5.24\}\end{array}$ & $\begin{array}{r}47.84 \\
\{5.48\}\end{array}$ \\
\hline Predicted Democratic & $\begin{array}{l}45.42 \\
\{5.87\}\end{array}$ & $\begin{array}{l}48.95 \\
\{5.91\}\end{array}$ & $\begin{array}{r}45.71 \\
\{5.59\}\end{array}$ & $\begin{array}{r}49.01 \\
\{5.61\}\end{array}$ & $\begin{array}{r}46.55 \\
\{5.19\}\end{array}$ & $\begin{array}{r}49.31 \\
\{5.22\}\end{array}$ \\
\hline Republican error & $\begin{array}{l}-3.48 \\
\{3.48\}\end{array}$ & $\begin{array}{c}0.03 \\
\{3.36\}\end{array}$ & $\begin{array}{c}-3.18 \\
\{3.31\}\end{array}$ & $\begin{array}{c}0.10 \\
\{3.21\}\end{array}$ & $\begin{array}{l}-3.17 \\
\{2.67\}\end{array}$ & $\begin{array}{l}-0.47 \\
\{2.49\}\end{array}$ \\
\hline Democratic error & $\begin{array}{l}-4.57 \\
\{4.00\}\end{array}$ & $\begin{array}{l}-1.04 \\
\{3.45\}\end{array}$ & $\begin{array}{l}-4.27 \\
\{3.79\}\end{array}$ & $\begin{array}{l}-0.96 \\
\{3.29\}\end{array}$ & $\begin{array}{c}-3.19 \\
\{3.02\}\end{array}$ & $\begin{array}{l}-0.43 \\
\{2.70\}\end{array}$ \\
\hline Standardized & -1.80 & 0.02 & -1.63 & 0.07 & -1.59 & -0.22 \\
\hline Republican error & $(0.04)$ & $(0.04)$ & $(0.04)$ & $(0.04)$ & $(0.05)$ & $(0.05)$ \\
\hline Variance of stand'd & 3.32 & 3.07 & 2.82 & 2.69 & 1.86 & 1.58 \\
\hline Republican error & $(0.16)$ & $(0.16)$ & $(0.12)$ & $(0.14)$ & $(0.12)$ & $(0.10)$ \\
\hline Standardized & -2.38 & -0.55 & -2.22 & -0.51 & -1.63 & -0.23 \\
\hline Democratic error & $(0.05)$ & $(0.04)$ & $(0.05)$ & $(0.04)$ & $(0.06)$ & $(0.05)$ \\
\hline Variance of stand'd & 4.38 & 3.20 & 3.91 & 2.84 & 2.37 & 1.89 \\
\hline Democratic error & $(0.19)$ & $(0.14)$ & $(0.20)$ & $(0.13)$ & $(0.15)$ & $(0.12)$ \\
\hline Republican victory & 38.40 & & 38.93 & & 40.77 & \\
\hline Democratic victory & 61.60 & & 61.07 & & 59.23 & \\
\hline $\begin{array}{l}\text { Republican victory } \\
\text { predicted }\end{array}$ & 40.01 & & 40.22 & & 38.64 & \\
\hline $\begin{array}{l}\text { Democratic victory } \\
\text { predicted }\end{array}$ & 55.57 & & 55.15 & & 56.53 & \\
\hline Mispredicted victor & 20.73 & & 20.46 & & 19.18 & \\
\hline Mispredicted victor & 24.23 & & 24.26 & & 28.41 & \\
\hline & & & e Obsert & n Per $\mathrm{P}$ & & \\
\hline & & & & & & \\
\hline Republican share & $\begin{array}{c}50.01 \\
\{8.97\}\end{array}$ & & $\begin{array}{c}49.68 \\
\{8.72\}\end{array}$ & & $\begin{array}{c}50.11 \\
\{8.02\}\end{array}$ & \\
\hline Democratic share & $\begin{array}{r}47.69 \\
\{8.92\}\end{array}$ & & $\begin{array}{l}48.09 \\
\{8.53\}\end{array}$ & & $\begin{array}{r}47.65 \\
\{7.85\}\end{array}$ & \\
\hline Republican victory & 53.15 & & 52.21 & & 53.85 & \\
\hline Democratic victory & 46.85 & & 47.79 & & 46.15 & \\
\hline $\begin{array}{l}\text { Mispredicted victor } \\
\text { using prior race }\end{array}$ & 16.08 & & 16.18 & & 19.66 & \\
\hline
\end{tabular}


Web Appendix Table 6: Pre-Election Polls, by Year

\begin{tabular}{|c|c|c|c|c|c|c|}
\hline \multirow[b]{2}{*}{ Republican share } & \multicolumn{2}{|c|}{$\begin{array}{l}2000 \text { Polls } \\
\mathrm{N}=475\end{array}$} & \multicolumn{2}{|c|}{$\begin{array}{l}2004 \text { Polls } \\
\mathrm{N}=705\end{array}$} & \multicolumn{2}{|c|}{$\begin{array}{l}2008 \text { Polls } \\
\mathrm{N}=677\end{array}$} \\
\hline & $\begin{array}{c}\text { Raw } \\
46.88 \\
\{6.43\}\end{array}$ & Adj & $\begin{array}{c}\text { Raw } \\
50.37 \\
\{4.90\}\end{array}$ & Adj & $\begin{array}{c}\text { Raw } \\
46.80 \\
\{6.39\}\end{array}$ & Adj \\
\hline Democratic share & $\begin{array}{r}49.37 \\
\{6.08\}\end{array}$ & & $\begin{array}{l}48.69 \\
\{4.80\}\end{array}$ & & $\begin{array}{r}51.78 \\
\{6.44\}\end{array}$ & \\
\hline Predicted Republican & $\begin{array}{l}42.86 \\
\{6.15\}\end{array}$ & $\begin{array}{c}47.12 \\
\{6.70\}\end{array}$ & $\begin{array}{l}46.51 \\
\{5.23\}\end{array}$ & $\begin{array}{r}49.63 \\
\{5.59\}\end{array}$ & $\begin{array}{l}44.10 \\
\{6.13\}\end{array}$ & $\begin{array}{l}47.47 \\
\{6.47\}\end{array}$ \\
\hline Predicted Democratic & $\begin{array}{l}43.38 \\
\{6.00\}\end{array}$ & $\begin{array}{l}47.62 \\
\{6.01\}\end{array}$ & $\begin{array}{l}45.37 \\
\{5.08\}\end{array}$ & $\begin{array}{c}48.38 \\
\{5.14\}\end{array}$ & $\begin{array}{l}46.91 \\
\{6.10\}\end{array}$ & $\begin{array}{r}50.47 \\
\{6.25\}\end{array}$ \\
\hline Republican error & $\begin{array}{l}-4.02 \\
\{3.74\}\end{array}$ & $\begin{array}{c}0.24 \\
\{3.64\}\end{array}$ & $\begin{array}{l}-3.86 \\
\{3.02\}\end{array}$ & $\begin{array}{c}-0.74 \\
\{2.81\}\end{array}$ & $\begin{array}{l}-2.70 \\
\{3.60\}\end{array}$ & $\begin{array}{c}0.67 \\
\{3.52\}\end{array}$ \\
\hline Democratic error & $\begin{array}{l}-5.99 \\
\{4.54\}\end{array}$ & $\begin{array}{l}-1.75 \\
\{4.07\}\end{array}$ & $\begin{array}{c}-3.32 \\
\{3.02\}\end{array}$ & $\begin{array}{c}-0.31 \\
\{2.71\}\end{array}$ & $\begin{array}{l}-4.87 \\
\{4.11\}\end{array}$ & $\begin{array}{l}-1.31 \\
\{3.53\}\end{array}$ \\
\hline $\begin{array}{l}\text { Standardized } \\
\text { Republican error }\end{array}$ & $\begin{array}{l}-1.98 \\
(0.09)\end{array}$ & $\begin{array}{c}0.14 \\
(0.08)\end{array}$ & $\begin{array}{l}-2.07 \\
(0.06)\end{array}$ & $\begin{array}{l}-0.40 \\
(0.06)\end{array}$ & $\begin{array}{l}-1.39 \\
(0.07)\end{array}$ & $\begin{array}{c}0.37 \\
(0.08)\end{array}$ \\
\hline Variance of stand'd & 3.47 & 3.34 & 2.86 & 2.45 & 3.43 & 3.23 \\
\hline Republican error & $(0.27)$ & $(0.33)$ & $(0.27)$ & $(0.26)$ & $(0.23)$ & $(0.19)$ \\
\hline Standardized & -3.01 & -0.90 & -1.78 & -0.17 & -2.55 & -0.70 \\
\hline Democratic error & $(0.11)$ & $(0.09)$ & $(0.06)$ & $(0.05)$ & $(0.07)$ & $(0.07)$ \\
\hline Variance of stand'd & 5.55 & 4.17 & 2.69 & 2.13 & 4.64 & 3.38 \\
\hline Democratic error & $(0.49)$ & $(0.31)$ & $(0.17)$ & $(0.16)$ & $(0.28)$ & $(0.21)$ \\
\hline Republican victory & 43.58 & & 49.93 & & 22.75 & \\
\hline Democratic victory & 56.42 & & 50.07 & & 77.25 & \\
\hline $\begin{array}{l}\text { Republican victory } \\
\text { predicted }\end{array}$ & 43.58 & & 45.53 & & 31.76 & \\
\hline $\begin{array}{l}\text { Democratic victory } \\
\text { predicted }\end{array}$ & 52.84 & & 47.52 & & 65.88 & \\
\hline Mispredicted victor & 19.58 & & 26.95 & & 15.07 & \\
\hline Mispredicted victor & 26.95 & & 12.91 & & 34.12 & \\
\hline & & & Obser & n Per $\mathrm{B}$ & & \\
\hline & & & & & & \\
\hline Republican share & $\begin{array}{r}49.90 \\
\{8.71\}\end{array}$ & & $\begin{array}{r}52.36 \\
\{8.28\}\end{array}$ & & $\begin{array}{r}47.97 \\
\{9.48\}\end{array}$ & \\
\hline Democratic share & $\begin{array}{r}45.94 \\
\{8.32\}\end{array}$ & & $\begin{array}{l}46.47 \\
\{8.28\}\end{array}$ & & $\begin{array}{c}50.46 \\
\{9.50\}\end{array}$ & \\
\hline Republican victory & 57.45 & & 58.70 & & 44.00 & \\
\hline Democratic victory & 42.55 & & 41.30 & & 56.00 & \\
\hline $\begin{array}{l}\text { Mispredicted victor } \\
\text { using prior race }\end{array}$ & 23.40 & & 6.52 & & 18.00 & \\
\hline
\end{tabular}

"Adj" means treating undecided respondents as strongly ignorable. The standardized prediction errors are calculated using the equation in the text. Under the null that the poll results are i.i.d. draws from the true distribution, the mean of the standardized prediction error is 0 and the variance is 1 . Prediction errors and shares are in units of percentage points. Standard deviations in braces. Standard errors in parentheses. Standard errors on variance estimates are bootstrapped with 1000 repetitions. 
Web Appendix Table 7: Error in Pre-Election Polls, by Inclusion of Third Party Candidates

\begin{tabular}{|c|c|c|c|c|c|c|c|}
\hline & \multicolumn{3}{|c|}{ Republican Prediction Error } & \multicolumn{3}{|c|}{ Democratic Prediction Error } & \multirow[b]{2}{*}{$\begin{array}{l}\text { Number } \\
\text { of Polls }\end{array}$} \\
\hline & Adj & Stand'd & $\begin{array}{l}\text { Stand'd } \\
\text { Var. }\end{array}$ & Adj & Stand'd & $\begin{array}{l}\text { Stand'd } \\
\text { Var. }\end{array}$ & \\
\hline All 2000 polls & $\begin{array}{c}0.24 \\
(0.17)\end{array}$ & $\begin{array}{c}0.14 \\
(0.19)\end{array}$ & $\begin{array}{c}3.34 \\
(0.33)\end{array}$ & $\begin{array}{l}-1.75 \\
(0.08)\end{array}$ & $\begin{array}{l}-0.90 \\
(0.09)\end{array}$ & $\begin{array}{c}4.17 \\
(0.32)\end{array}$ & 475 \\
\hline Buchanan included & $\begin{array}{l}-0.15 \\
(0.20)\end{array}$ & $\begin{array}{l}-0.07 \\
(0.24)\end{array}$ & $\begin{array}{c}2.77 \\
(0.27)\end{array}$ & $\begin{array}{l}-2.08 \\
(0.10)\end{array}$ & $\begin{array}{l}-1.06 \\
(0.12)\end{array}$ & $\begin{array}{l}4.03 \\
(0.34)\end{array}$ & 292 \\
\hline Buchanan not included & $\begin{array}{c}0.87 \\
(0.30)\end{array}$ & $\begin{array}{c}0.47 \\
(0.30)\end{array}$ & $\begin{array}{c}4.08 \\
(0.68)\end{array}$ & $\begin{array}{l}-1.22 \\
(0.15)\end{array}$ & $\begin{array}{l}-0.64 \\
(0.15)\end{array}$ & $\begin{array}{c}4.32 \\
(0.58)\end{array}$ & 183 \\
\hline Nader included & $\begin{array}{c}0.00 \\
(0.16)\end{array}$ & $\begin{array}{c}0.01 \\
(0.20)\end{array}$ & $\begin{array}{c}2.63 \\
(0.26)\end{array}$ & $\begin{array}{l}-2.03 \\
(0.08)\end{array}$ & $\begin{array}{l}-1.05 \\
(0.10)\end{array}$ & $\begin{array}{c}3.87 \\
(0.32)\end{array}$ & 393 \\
\hline Nader not included & $\begin{array}{l}1.41 \\
(0.56)\end{array}$ & $\begin{array}{c}0.75 \\
(0.50)\end{array}$ & $\begin{array}{c}6.35 \\
(1.27)\end{array}$ & $\begin{array}{l}-0.40 \\
(0.28)\end{array}$ & $\begin{array}{l}-0.20 \\
(0.25)\end{array}$ & $\begin{array}{c}5.10 \\
(0.94)\end{array}$ & 82 \\
\hline $\begin{array}{l}\text { Both Buchanan } \\
\text { and Nader included }\end{array}$ & $\begin{array}{l}-0.13 \\
(0.20)\end{array}$ & $\begin{array}{l}-0.06 \\
(0.24)\end{array}$ & $\begin{array}{c}2.74 \\
(0.27)\end{array}$ & $\begin{array}{l}-2.15 \\
(0.10)\end{array}$ & $\begin{array}{l}-1.10 \\
(0.12)\end{array}$ & $\begin{array}{c}3.96 \\
(0.35)\end{array}$ & 277 \\
\hline $\begin{array}{l}\text { Any third party } \\
\text { candidate included }\end{array}$ & $\begin{array}{l}-0.09 \\
(0.16)\end{array}$ & $\begin{array}{l}-0.03 \\
(0.19)\end{array}$ & $\begin{array}{c}2.70 \\
(0.25)\end{array}$ & $\begin{array}{l}-1.88 \\
(0.08)\end{array}$ & $\begin{array}{l}-0.97 \\
(0.10)\end{array}$ & $\begin{array}{c}3.97 \\
(0.32)\end{array}$ & 434 \\
\hline $\begin{array}{l}\text { No third party } \\
\text { candidate included }\end{array}$ & $\begin{array}{c}3.74 \\
(0.80)\end{array}$ & $\begin{array}{l}1.92 \\
(0.74)\end{array}$ & $\begin{array}{c}6.80 \\
(1.66)\end{array}$ & $\begin{array}{l}-0.38 \\
(0.41)\end{array}$ & $\begin{array}{l}-0.16 \\
(0.38)\end{array}$ & $\begin{array}{c}5.87 \\
(1.62)\end{array}$ & 41 \\
\hline All 2004 polls & $\begin{array}{l}-0.74 \\
(0.11)\end{array}$ & $\begin{array}{l}-0.40 \\
(0.10)\end{array}$ & $\begin{array}{c}2.45 \\
(0.26)\end{array}$ & $\begin{array}{l}-0.31 \\
(0.06)\end{array}$ & $\begin{array}{l}-0.17 \\
(0.05)\end{array}$ & $\begin{array}{c}2.13 \\
(0.15)\end{array}$ & 705 \\
\hline Nader included & $\begin{array}{l}-0.76 \\
(0.14)\end{array}$ & $\begin{array}{l}-0.42 \\
(0.13)\end{array}$ & $\begin{array}{c}2.54 \\
(0.34)\end{array}$ & $\begin{array}{l}-0.79 \\
(0.08)\end{array}$ & $\begin{array}{l}-0.44 \\
(0.07)\end{array}$ & $\begin{array}{c}2.08 \\
(0.18)\end{array}$ & 391 \\
\hline Nader not included & $\begin{array}{l}-0.72 \\
(0.16)\end{array}$ & $\begin{array}{l}-0.38 \\
(0.15)\end{array}$ & $\begin{array}{c}2.34 \\
(0.37)\end{array}$ & $\begin{array}{c}0.29 \\
(0.09)\end{array}$ & $\begin{array}{c}0.16 \\
(0.08)\end{array}$ & $\begin{array}{c}2.00 \\
(0.25)\end{array}$ & 314 \\
\hline $\begin{array}{l}\text { Any third party } \\
\text { candidate included }\end{array}$ & $\begin{array}{l}-0.92 \\
(0.12)\end{array}$ & $\begin{array}{l}-0.51 \\
(0.11)\end{array}$ & $\begin{array}{c}2.57 \\
(0.31)\end{array}$ & $\begin{array}{l}-0.57 \\
(0.07)\end{array}$ & $\begin{array}{l}-0.30 \\
(0.06)\end{array}$ & $\begin{array}{c}2.16 \\
(0.19)\end{array}$ & 567 \\
\hline $\begin{array}{l}\text { No third party } \\
\text { candidate included }\end{array}$ & $\begin{array}{l}-0.03 \\
(0.21)\end{array}$ & $\begin{array}{c}0.02 \\
(0.21)\end{array}$ & $\begin{array}{c}1.72 \\
(0.22)\end{array}$ & $\begin{array}{c}0.75 \\
(0.11)\end{array}$ & $\begin{array}{c}0.38 \\
(0.11)\end{array}$ & $\begin{array}{l}1.67 \\
(0.21)\end{array}$ & 138 \\
\hline All 2008 polls & $\begin{array}{c}0.67 \\
(0.14)\end{array}$ & $\begin{array}{c}0.37 \\
(0.14)\end{array}$ & $\begin{array}{c}3.23 \\
(0.19)\end{array}$ & $\begin{array}{l}-1.31 \\
(0.07)\end{array}$ & $\begin{array}{l}-0.70 \\
(0.07)\end{array}$ & $\begin{array}{c}3.38 \\
(0.20)\end{array}$ & 677 \\
\hline $\begin{array}{l}\text { Any third party } \\
\text { candidate included }\end{array}$ & $\begin{array}{c}0.04 \\
(0.15)\end{array}$ & $\begin{array}{c}0.07 \\
(0.16)\end{array}$ & $\begin{array}{c}2.93 \\
(0.23)\end{array}$ & $\begin{array}{l}-1.58 \\
(0.08)\end{array}$ & $\begin{array}{l}-0.87 \\
(0.08)\end{array}$ & $\begin{array}{c}3.33 \\
(0.27)\end{array}$ & 472 \\
\hline $\begin{array}{l}\text { No third party } \\
\text { candidate included }\end{array}$ & $\begin{array}{c}2.13 \\
(0.25)\end{array}$ & $\begin{array}{l}1.05 \\
(0.26)\end{array}$ & $\begin{array}{c}3.26 \\
(0.32)\end{array}$ & $\begin{array}{l}-0.68 \\
(0.13)\end{array}$ & $\begin{array}{l}-0.31 \\
(0.13)\end{array}$ & $\begin{array}{c}3.30 \\
(0.32)\end{array}$ & 205 \\
\hline
\end{tabular}

All columns treat undecided respondents as strongly ignorable. Under the null that the poll results are i.i.d. draws from the true distribution, the mean of the standardized prediction error is 0 and the variance is 1 . Prediction errors and shares are in units of percentage points. Third party candidates received $1.3 \%$ of the popular vote in $2008,1.0 \%$ in 2004 and $3.7 \%$ in 2000. Standard errors in parentheses. Standard errors on variances are bootstrapped with 1000 repetitions. 
Web Appendix Table 8: The Relation Between Forecast Errors and Prior Information

\begin{tabular}{|c|c|c|c|c|c|c|c|c|}
\hline & \multicolumn{8}{|c|}{ Dependent Variable $=2008$ Polls } \\
\hline & \multicolumn{4}{|c|}{ Republican Candidate } & \multicolumn{4}{|c|}{ Democratic Candidate } \\
\hline & (1) & $(2)$ & $(3)$ & (4) & (1) & $(2)$ & $(3)$ & (4) \\
\hline 2008 Outcome & $\begin{array}{c}0.861 \\
(0.041)\end{array}$ & & $\begin{array}{c}0.569 \\
(0.091)\end{array}$ & $\begin{array}{c}0.571 \\
(0.104)\end{array}$ & $\begin{array}{c}0.821 \\
(0.041)\end{array}$ & & $\begin{array}{c}0.507 \\
(0.085)\end{array}$ & $\begin{array}{c}0.492 \\
(0.099)\end{array}$ \\
\hline 2004 Outcome & & $\begin{array}{c}0.899 \\
(0.053)\end{array}$ & $\begin{array}{c}0.338 \\
(0.105)\end{array}$ & $\begin{array}{c}0.440 \\
(0.215)\end{array}$ & & $\begin{array}{c}0.855 \\
(0.045)\end{array}$ & $\begin{array}{c}0.360 \\
(0.090)\end{array}$ & $\begin{array}{c}0.500 \\
(0.154)\end{array}$ \\
\hline 2000 Outcome & & & & $\begin{array}{l}-0.205 \\
(0.114)\end{array}$ & & & & $\begin{array}{l}-0.144 \\
(0.106)\end{array}$ \\
\hline 1996 Outcome & & & & $\begin{array}{c}0.130 \\
(0.076)\end{array}$ & & & & $\begin{array}{c}0.023 \\
(0.135)\end{array}$ \\
\hline Constant & $\begin{array}{c}7.166 \\
(1.978)\end{array}$ & $\begin{array}{c}0.908 \\
(2.595)\end{array}$ & $\begin{array}{c}3.289 \\
(2.159)\end{array}$ & $\begin{array}{c}2.716 \\
(2.260)\end{array}$ & $\begin{array}{c}7.967 \\
(2.098)\end{array}$ & $\begin{array}{l}10.108 \\
(2.250)\end{array}$ & $\begin{array}{c}7.222 \\
(1.756)\end{array}$ & $\begin{array}{c}7.007 \\
(2.591)\end{array}$ \\
\hline \multirow{4}{*}{$\begin{array}{l}\text { R-squared } \\
\mathrm{N}=677\end{array}$} & 0.723 & 0.690 & 0.738 & 0.741 & 0.715 & 0.692 & 0.733 & 0.736 \\
\hline & \multicolumn{8}{|c|}{ Dependent Variable $=2004$ Polls } \\
\hline & \multicolumn{4}{|c|}{ Republican Candidate } & \multicolumn{4}{|c|}{ Democratic Candidate } \\
\hline & (1) & (2) & $(3)$ & (4) & (1) & (2) & $(3)$ & (4) \\
\hline 2004 Outcome & $\begin{array}{c}0.986 \\
(0.032)\end{array}$ & & $\begin{array}{c}0.927 \\
(0.122)\end{array}$ & $\begin{array}{c}0.951 \\
(0.131)\end{array}$ & $\begin{array}{c}0.915 \\
(0.032)\end{array}$ & & $\begin{array}{c}0.886 \\
(0.099)\end{array}$ & $\begin{array}{c}0.881 \\
(0.104)\end{array}$ \\
\hline 2000 Outcome & & $\begin{array}{c}0.927 \\
(0.089)\end{array}$ & $\begin{array}{c}0.061 \\
(0.119)\end{array}$ & $\begin{array}{c}0.143 \\
(0.093)\end{array}$ & & $\begin{array}{c}0.828 \\
(0.111)\end{array}$ & $\begin{array}{c}0.033 \\
(0.103)\end{array}$ & $\begin{array}{c}0.006 \\
(0.128)\end{array}$ \\
\hline 1996 Outcome & & & & $\begin{array}{l}-0.139 \\
(0.159)\end{array}$ & & & & $\begin{array}{c}0.043 \\
(0.137)\end{array}$ \\
\hline Constant & $\begin{array}{c}-0.034 \\
(1.567)\end{array}$ & $\begin{array}{c}5.125 \\
(4.268)\end{array}$ & $\begin{array}{l}-0.006 \\
(1.616)\end{array}$ & $\begin{array}{c}0.456 \\
(1.540)\end{array}$ & $\begin{array}{c}3.851 \\
(1.643)\end{array}$ & $\begin{array}{c}8.480 \\
(5.447)\end{array}$ & $\begin{array}{c}3.666 \\
(1.700)\end{array}$ & $\begin{array}{c}3.095 \\
(2.472)\end{array}$ \\
\hline \multirow{4}{*}{$\begin{array}{l}\text { R-squared } \\
\mathrm{N}=705\end{array}$} & 0.747 & 0.681 & 0.747 & 0.750 & 0.729 & 0.582 & 0.730 & 0.730 \\
\hline & \multicolumn{8}{|c|}{ Dependent Variable $=2000$ Polls } \\
\hline & \multicolumn{4}{|c|}{ Republican Candidate } & \multicolumn{4}{|c|}{ Democratic Candidate } \\
\hline & (1) & $(2)$ & (3) & & (1) & $(2)$ & (3) & \\
\hline 2000 Outcome & $\begin{array}{c}0.883 \\
(0.049)\end{array}$ & & $\begin{array}{c}0.745 \\
(0.081)\end{array}$ & & $\begin{array}{c}0.764 \\
(0.047)\end{array}$ & & $\begin{array}{c}0.594 \\
(0.143)\end{array}$ & \\
\hline 1996 Outcome & & $\begin{array}{c}1.021 \\
(0.072)\end{array}$ & $\begin{array}{c}0.185 \\
(0.093)\end{array}$ & & & $\begin{array}{c}0.932 \\
(0.059)\end{array}$ & $\begin{array}{c}0.228 \\
(0.159)\end{array}$ & \\
\hline Constant & $\begin{array}{c}5.719 \\
(2.213)\end{array}$ & $\begin{array}{c}6.574 \\
(2.726)\end{array}$ & $\begin{array}{c}4.834 \\
(2.298)\end{array}$ & & $\begin{array}{c}9.920 \\
(2.399)\end{array}$ & $\begin{array}{c}1.090 \\
(3.067)\end{array}$ & $\begin{array}{c}6.889 \\
(2.467)\end{array}$ & \\
\hline $\begin{array}{l}\text { R-squared } \\
\mathrm{N}=475\end{array}$ & 0.717 & 0.637 & 0.721 & & 0.598 & 0.558 & 0.602 & \\
\hline
\end{tabular}


Web Appendix Table 9: Descriptive Statistics of Manski Poll

\begin{tabular}{|c|c|c|c|c|}
\hline \multirow[b]{3}{*}{ Number of resnondents } & \multicolumn{2}{|c|}{ Wave 1} & \multicolumn{2}{|c|}{ Wave 2} \\
\hline & Probabilistic & Control & Probabilistic & Control \\
\hline & 647 & 682 & 675 & 711 \\
\hline $\begin{array}{l}\text { Fraction expressing no uncertainty } \\
\text { in candidate preference }\end{array}$ & 0.764 & & 0.767 & \\
\hline $\begin{array}{l}\text { Fraction expressing little }(<10 \%) \\
\text { uncertainty in candidate preference }\end{array}$ & 0.897 & & 0.908 & \\
\hline \multicolumn{5}{|l|}{ Probability of voting } \\
\hline Mean & 0.841 & 0.839 & 0.857 & 0.857 \\
\hline Standard deviation & 0.338 & 0.368 & 0.315 & 0.351 \\
\hline 10th percentile & 0 & 0 & 0.2 & 0 \\
\hline 25th percentile & 0.99 & 1 & 0.99 & 1 \\
\hline 50th percentile & 1 & 1 & 1 & 1 \\
\hline \multicolumn{5}{|l|}{ Demographics } \\
\hline Age & $\begin{array}{c}47.209 \\
\{16.908\}\end{array}$ & $\begin{array}{c}47.443 \\
\{16.744\}\end{array}$ & $\begin{array}{c}47.108 \\
\{16.940\}\end{array}$ & $\begin{array}{r}47.498 \\
\{17.701\}\end{array}$ \\
\hline White & 0.810 & 0.792 & 0.796 & 0.788 \\
\hline Male & 0.488 & 0.493 & 0.484 & 0.498 \\
\hline Household head & 0.819 & 0.833 & 0.839 & 0.826 \\
\hline Married & 0.603 & 0.589 & 0.582 & 0.536 \\
\hline Metro area & 0.807 & 0.826 & 0.847 & 0.840 \\
\hline Employed & 0.621 & 0.572 & 0.573 & 0.589 \\
\hline Less than high school & 0.130 & 0.166 & 0.166 & 0.166 \\
\hline High school graduate & 0.272 & 0.224 & 0.273 & 0.276 \\
\hline Some college or associate degree & 0.332 & 0.359 & 0.289 & 0.293 \\
\hline B.A. or higher & 0.266 & 0.251 & 0.273 & 0.266 \\
\hline Northeast & 0.176 & 0.188 & 0.188 & 0.173 \\
\hline Midwest & 0.283 & 0.249 & 0.276 & 0.294 \\
\hline South & 0.331 & 0.331 & 0.313 & 0.329 \\
\hline West & 0.210 & 0.232 & 0.224 & 0.204 \\
\hline F-statistic from joint test of significance & 1.12 & & 0.54 & \\
\hline $\mathrm{p}$-value from joint test of significance & 0.3393 & & 0.8987 & \\
\hline
\end{tabular}

Standard deviations in braces. 


\begin{tabular}{|c|c|c|c|c|c|c|}
\hline & \multicolumn{3}{|c|}{ Probabilistic Group } & \multicolumn{3}{|c|}{ Control Group } \\
\hline & Bush & Kerry & Other & Bush & Kerry & Other \\
\hline Wave 1 & \multicolumn{3}{|c|}{$\mathrm{N}=647$} & \multicolumn{3}{|c|}{$\mathrm{N}=682$} \\
\hline Survey weighted & $\begin{array}{l}46.534 \\
(2.137)\end{array}$ & $\begin{array}{l}49.873 \\
(2.157)\end{array}$ & $\begin{array}{c}3.593 \\
(0.751)\end{array}$ & $\begin{array}{l}47.190 \\
(2.276)\end{array}$ & $\begin{array}{l}49.551 \\
(2.290)\end{array}$ & $\begin{array}{c}3.259 \\
(0.823)\end{array}$ \\
\hline $\mathrm{P}($ vote $)>0$ & \multicolumn{3}{|c|}{$\mathrm{N}=577$} & \multicolumn{3}{|c|}{$\mathrm{N}=572$} \\
\hline Survey weighted & $\begin{array}{l}48.919 \\
(2.318)\end{array}$ & $\begin{array}{l}48.078 \\
(2.336)\end{array}$ & $\begin{array}{c}3.004 \\
(0.750)\end{array}$ & $\begin{array}{l}48.806 \\
(2.487)\end{array}$ & $\begin{array}{l}48.900 \\
(2.496)\end{array}$ & $\begin{array}{c}2.293 \\
(0.840)\end{array}$ \\
\hline $\begin{array}{l}\text { Above, and } \\
\text { participation weighted }\end{array}$ & $\begin{array}{l}48.915 \\
(2.403)\end{array}$ & $\begin{array}{l}48.410 \\
(2.415)\end{array}$ & $\begin{array}{c}2.675 \\
(0.637)\end{array}$ & & & \\
\hline $\begin{array}{l}\text { Above, and } \\
\text { missing data weighted } \\
\text { p-values }\end{array}$ & $\begin{array}{l}48.585 \\
(2.437)\end{array}$ & $\begin{array}{l}48.761 \\
(2.455)\end{array}$ & $\begin{array}{c}2.654 \\
(0.628)\end{array}$ & $\begin{array}{l}48.763 \\
(2.490)\end{array}$ & $\begin{array}{l}48.949 \\
(2.499)\end{array}$ & $\begin{array}{l}2.288 \\
(0.839)\end{array}$ \\
\hline \multirow{3}{*}{\multicolumn{2}{|c|}{$\begin{array}{l}\operatorname{Bush}(M=1)=\operatorname{Bush}(M=0) \\
\operatorname{Kerry}(M=1)=\operatorname{Kerry}(M=0) \\
\text { Joint }\end{array}$}} & 0.9593 & & & & \\
\hline & & 0.9573 & & & & \\
\hline & & 0.9445 & & & & \\
\hline Wave 2 & \multicolumn{3}{|c|}{$\mathrm{N}=675$} & \multicolumn{3}{|c|}{$\mathrm{N}=711$} \\
\hline $\begin{array}{l}\text { Survey weighted } \\
\qquad \mathrm{P}(\text { vote })>0\end{array}$ & $\begin{array}{l}45.528 \\
(2.069)\end{array}$ & $\begin{array}{c}50.997 \\
(2.061) \\
\mathrm{N}=613\end{array}$ & $\begin{array}{c}3.474 \\
(0.661)\end{array}$ & $\begin{array}{l}45.519 \\
(2.144)\end{array}$ & $\begin{array}{c}49.110 \\
(2.153) \\
\mathrm{N}=609\end{array}$ & $\begin{array}{c}5.371 \\
(1.093)\end{array}$ \\
\hline Survey weighted & $\begin{array}{l}45.037 \\
(2.173)\end{array}$ & $\begin{array}{l}52.117 \\
(2.173)\end{array}$ & $\begin{array}{l}2.846 \\
(0.647)\end{array}$ & $\begin{array}{l}47.435 \\
(2.337)\end{array}$ & $\begin{array}{l}49.507 \\
(2.341)\end{array}$ & $\begin{array}{c}3.058 \\
(0.931)\end{array}$ \\
\hline $\begin{array}{l}\text { Above, and } \\
\text { participation weighted }\end{array}$ & $\begin{array}{l}44.913 \\
(2.232)\end{array}$ & $\begin{array}{l}52.425 \\
(2.231)\end{array}$ & $\begin{array}{c}2.662 \\
(0.661)\end{array}$ & & & \\
\hline $\begin{array}{l}\text { Above, and } \\
\text { missing data weighted } \\
\text { p-values }\end{array}$ & $\begin{array}{l}44.772 \\
(2.237)\end{array}$ & $\begin{array}{l}52.567 \\
(2.238)\end{array}$ & $\begin{array}{c}2.661 \\
(0.656)\end{array}$ & $\begin{array}{l}47.408 \\
(2.338)\end{array}$ & $\begin{array}{l}49.551 \\
(2.342)\end{array}$ & $\begin{array}{c}3.042 \\
(0.924)\end{array}$ \\
\hline $\operatorname{Bush}(M=1)=\operatorname{Bush}(M=0)$ & & 0.4155 & & & & \\
\hline $\operatorname{Kerry}(M=1)=\operatorname{Kerry}(M=0$ & & 0.3518 & & & & \\
\hline Joint & & 0.6374 & & & & \\
\hline Wave 1 \& 2 Combined & & $\mathrm{N}=1322$ & & & $\mathrm{~N}=1393$ & \\
\hline $\begin{array}{l}\text { Survey weighted } \\
\qquad \mathrm{P}(\text { vote })>0\end{array}$ & $\begin{array}{l}46.037 \\
(1.485)\end{array}$ & $\begin{array}{c}50.429 \\
(1.490) \\
\mathrm{N}=1190\end{array}$ & $\begin{array}{c}3.534 \\
(0.501)\end{array}$ & $\begin{array}{l}46.364 \\
(1.563)\end{array}$ & $\begin{array}{c}49.333 \\
(1.573) \\
\mathrm{N}=1181\end{array}$ & $\begin{array}{c}4.303 \\
(0.684)\end{array}$ \\
\hline Survey weighted & $\begin{array}{l}46.973 \\
(1.582)\end{array}$ & $\begin{array}{l}50.102 \\
(1.589)\end{array}$ & $\begin{array}{l}2.925 \\
(0.495)\end{array}$ & $\begin{array}{l}48.119 \\
(1.705)\end{array}$ & $\begin{array}{l}49.204 \\
(1.709)\end{array}$ & $\begin{array}{c}2.676 \\
(0.627)\end{array}$ \\
\hline $\begin{array}{l}\text { Above, and } \\
\text { participation weighted }\end{array}$ & $\begin{array}{l}46.886 \\
(1.633)\end{array}$ & $\begin{array}{l}50.445 \\
(1.636)\end{array}$ & $\begin{array}{c}2.669 \\
(0.459)\end{array}$ & & & \\
\hline $\begin{array}{l}\text { Above, and } \\
\text { missing data weighted } \\
\text { p-values }\end{array}$ & $\begin{array}{l}46.655 \\
(1.646)\end{array}$ & $\begin{array}{l}50.687 \\
(1.652)\end{array}$ & $\begin{array}{c}2.657 \\
(0.454)\end{array}$ & $\begin{array}{l}48.084 \\
(1.706)\end{array}$ & $\begin{array}{l}49.250 \\
(1.711)\end{array}$ & $\begin{array}{c}2.666 \\
(0.624)\end{array}$ \\
\hline $\operatorname{Bush}(T=1)=\operatorname{Bush}(T=0)$ & & 0.5467 & & & & \\
\hline $\operatorname{Kerry}(\mathrm{T}=1)=\operatorname{Kerry}(\mathrm{T}=0)$ & & 0.5457 & & & & \\
\hline Joint & & 0.8295 & & & & \\
\hline
\end{tabular}

In all results we employ survey weights provided by TESS designed to match the demographics of the surveyed sample to the U.S. Census and the Knowledge Networks Panel. The pooled results employ DFL weights to account for differences in observed sample demographics between waves. Likely voter weights use the reported probability of voting (for the Probabilistic group only) to adjust results. The missing data weights use DFL weights to account for 58 dropped observations with missing poll results on observed dimensions of demographics. All weights (except the TESS survey weights) are estimated using probit regressions of the appropriate outcome on a flexible set of the individual demographics including age, age squared, and dummies for each of the categorical variables in web appendix Table 9. Actual national 2004 election results were Bush 50.733\%, Kerry $48.270 \%$, and Other $0.996 \%$. Heteroskedasticity robust standard errors in parentheses. 


\title{
Web Appendix: Figures
}

\author{
Elias Walsh, Sarah Dolfin and John DiNardo
}

January 30, 2009

\section{Web Appendix Figure 1: Density Estimates of Standardized Prediction Errors, by Election Year}

The figure displays a kernel density of the standardized prediction errors for presidential state races by election year. The vertical lines are the estimated mean associated with the appropriate density. In comparison to the standard normal density, the theoretical prediction under random probability sampling, the poll densities are more disperse and are not centered at 0 , indicating bias. The bandwidth for density estimation was chosen by ocular inspection and is 0.2 .

Web Appendix Figure 2: Density Estimates of Standardized Prediction Errors, by Poll Subgroup

The figure displays a kernel density of the standardized prediction errors for presidential state races by poll subgroup. In comparison to the standard normal density, the theoretical prediction under random probability sampling, the poll densities are more disperse, though the polls within two weeks of the election do show less dispersion. The bandwidth for density estimation was chosen by ocular inspection and is 0.2 .

Web Appendix Figures 3 \& 4: The Relation Between Forecasts and Election Results Each circle represents the mean of all poll results in a statewide election. The dashed line is the estimated line from a regression of the poll prediction on the actual election outcome. The solid line is the 45-degree line. The slope of the estimated line is always less than 1 (see also web appendix Table 8). Thus bias in polls tends to work in a way that understates larger vote shares and overstates smaller vote shares. This could be explained as a result of "honest Bayesian" type behavior on the part pollsters, or simply an artifact of other problems in polling that cause bias. For Democratic candidates the point at which the regression line crosses the 45 -degree line is below $50 \%$, while for Republicans this crossing point tends to be higher. If pollsters do act like "honest Bayesians" then these crossing points may be indicative of the pollsters' prior beliefs about a candidate's vote share. If for example, pollsters are reporting the maximum posterior density, then the nonzero intercept and departure of the slope from 1 are the consequence of the standard omitted variable calculations where the omitted variable is pollsters' prior information. These general findings are not changed much if we limit the analysis to only those polls conducted within two weeks of the election.

Web Appendix Figures 5 \& 6: The Relation Between Forecast Errors and Election Results Figures 5 and 6 are analogues to Figures 3 and 4 with poll prediction errors in the place of the predicted vote shares. Each circle represents the mean of all poll prediction errors in a statewide election. The estimated line from a regression of the poll prediction errors on the actual election outcome is always negative. The main benefit to displaying the prediction errors rather than the predicted shares is that the scatter plot is more clearly presented.

Web Appendix Figure 7: Distribution of Polls Across States By Election Result and Number of Electoral Votes

Each circle represents a statewide election. The area of the circle is proportional to the number of polls in that race. Races with more polls tend to be concentrated in states with more electoral votes and in states that are more highly contested. We would expect to see very large circles in states that both have many electoral votes and are close races, however, the only state with more than 40 electoral votes is California, a state that is not particularly competitive.

Web Appendix Figure 8: Standardized Prediction Errors Over Time

The figure displays scatter plots of standardized prediction errors for presidential statewide races and quantile 
regressions at the 10th, 50th, and 90th quantile. The lines in panels (a) and (c) present the results of a quantile regression of the prediction errors on the number of days before the election and a constant term. Panels (b) and (d) present the 10th and 90th quantiles, and associated confidence intervals from a designadaptive bandwidth quantile regression, limiting the sample to only those polls within 10 weeks of the election. The two dotted horizontal lines in each of panels (b) and (d) indicate the theoretical prediction of the 90th and 10th percentiles under standard normality (1.28/-1.28). The panels demonstrate that dispersion in the poll errors diminishes over time, but even for the closest polls to the election the dispersion exceeds that of a standard normal density.

Web Appendix Figures 9 \& 10: Standardized Prediction Errors Over Time, by Election Year

The figures display scatter plots of standardized prediction errors for presidential statewide races and quantile regressions at the 10th, 50th, and 90th quantile for polls separately by election year. The lines in panels (a), (c), and (e) present the results of a quantile regression of the prediction errors on the number of days before the election and a constant term. Panels (b), (d), and (f) present the 10th, and 90th quantiles and associated confidence intervals from a design-adaptive bandwidth quantile regression, limiting the sample to only those polls within 10 weeks of the election. The two dotted horizontal lines in each of panels (b), (d), and (f) indicate the theoretical prediction of the 90 th and 10th percentiles under standard normality $(1.28 /-1.28)$. As in Figure 9, the panels generally demonstrate that dispersion in the poll errors diminishes over time, but even for the closest polls to the election the dispersion exceeds that of a standard normal density. We see some variation across election, with the 2004 polls for both the Republican and Democratic candidate displaying more-or-less constant dispersion over time. Also, the design-adaptive bandwidth quantile regressions do not always reject the prediction for the 10th and 90th quantiles of the standard normal density for the closest polls to the election.

Web Appendix Figure 11: Density Estimates of Standardized Prediction Errors, by Detailed Poll Subgroup

Figure 11 is an extension of Figure 2 with two additional subgroups: polls that sum to 100-102 percentage points, and polls that do not allow third party candidates as an option for respondents. The polls that sum to 100-102 do not look much better than the density of all polls. The polls that exclude third parties show about the same amount of dispersion as polls more generally, but in the case of the Republican share the density is shifted to the right, indicating bias in the direction of over-prediction. The bandwidth for density estimation was chosen by ocular inspection and is 0.2 . 
Web Appendix Figure 1: Density Estimates of Standardized Prediction Errors, by Election Year

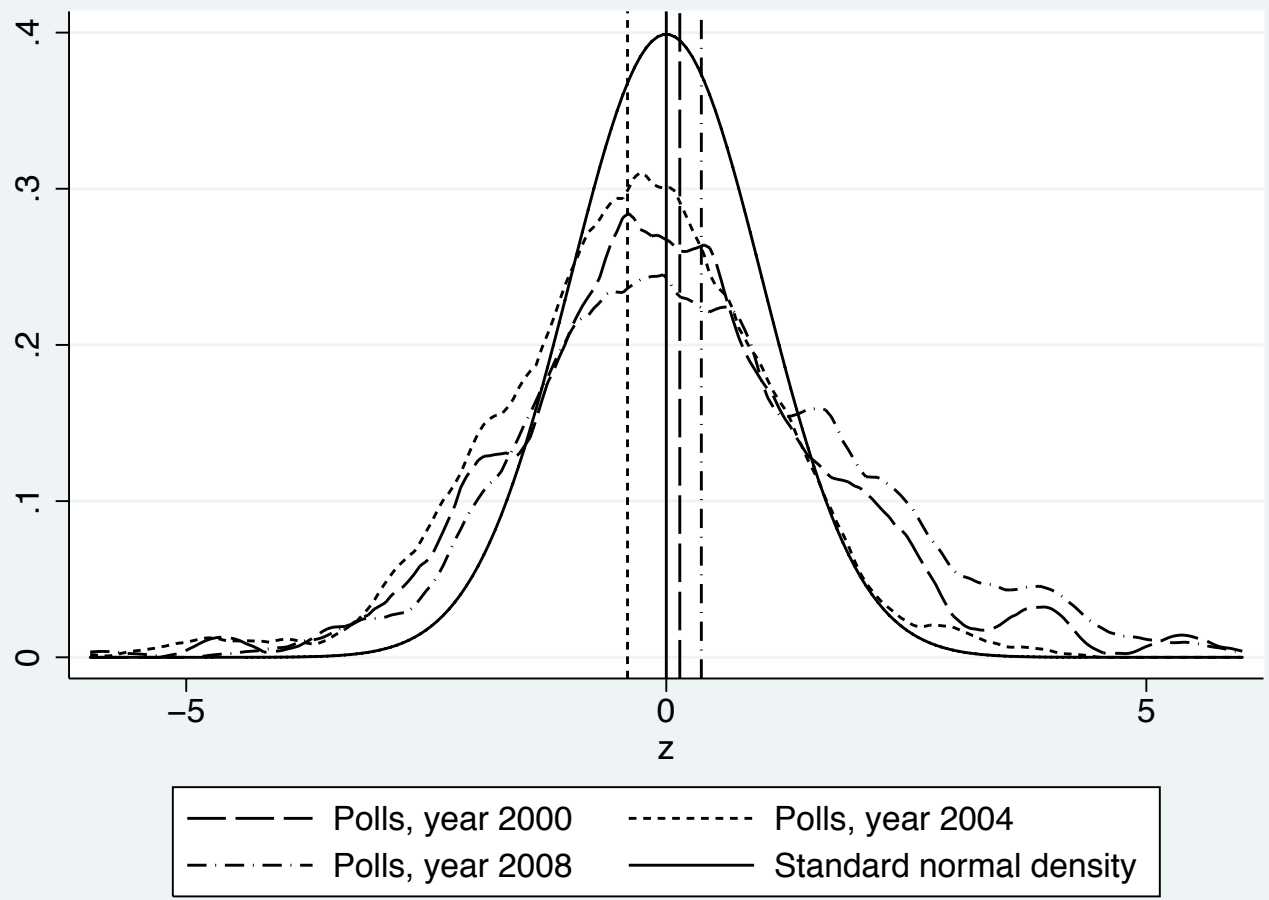

(a) Republican Prediction Error

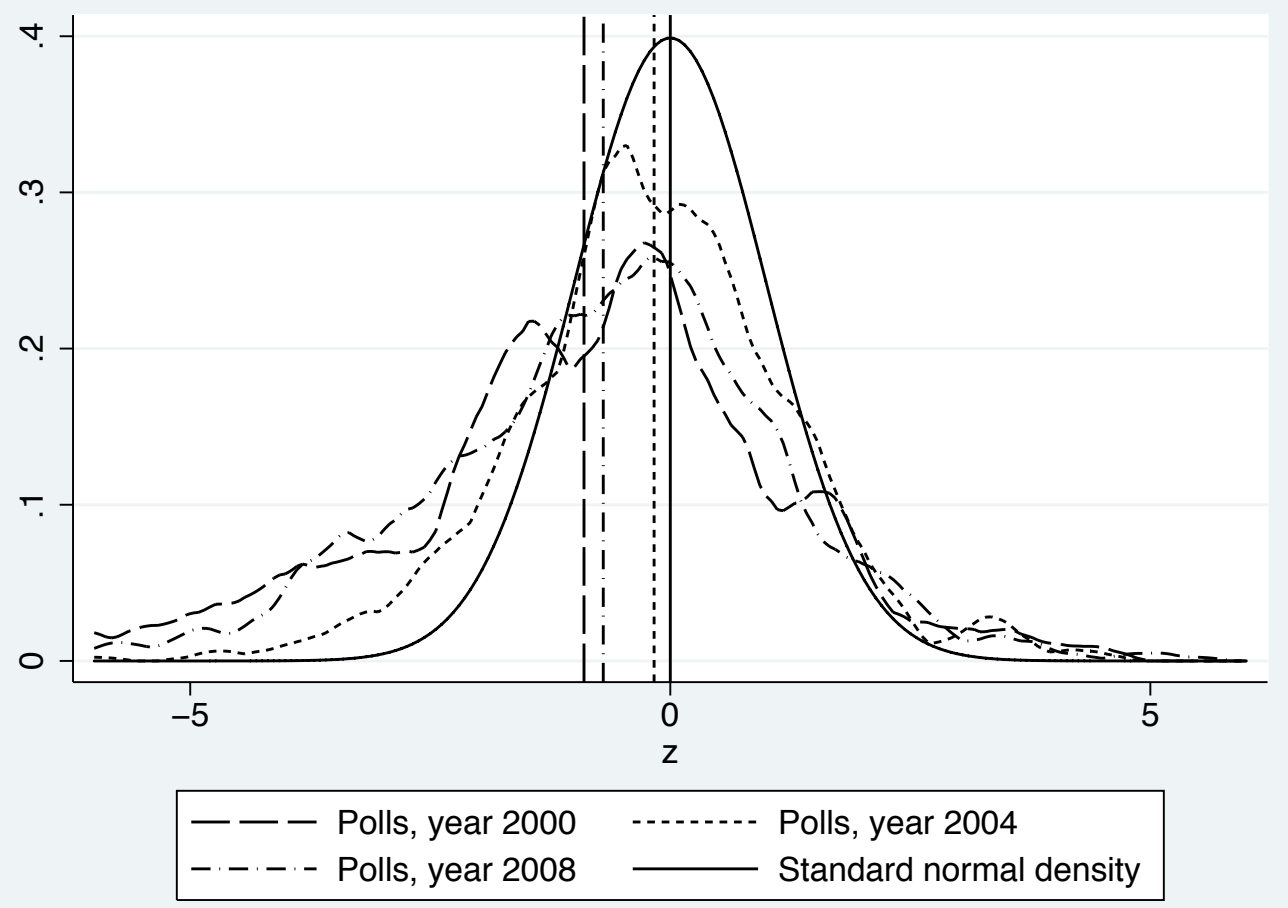

(b) Democratic Prediction Error

The figure displays a kernel density of the standardized prediction errors for presidential state races by election year. The vertical lines are the estimated mean associated with the appropriate density. 
Web Appendix Figure 2: Density Estimates of Standardized Prediction Errors, by Poll Subgroup

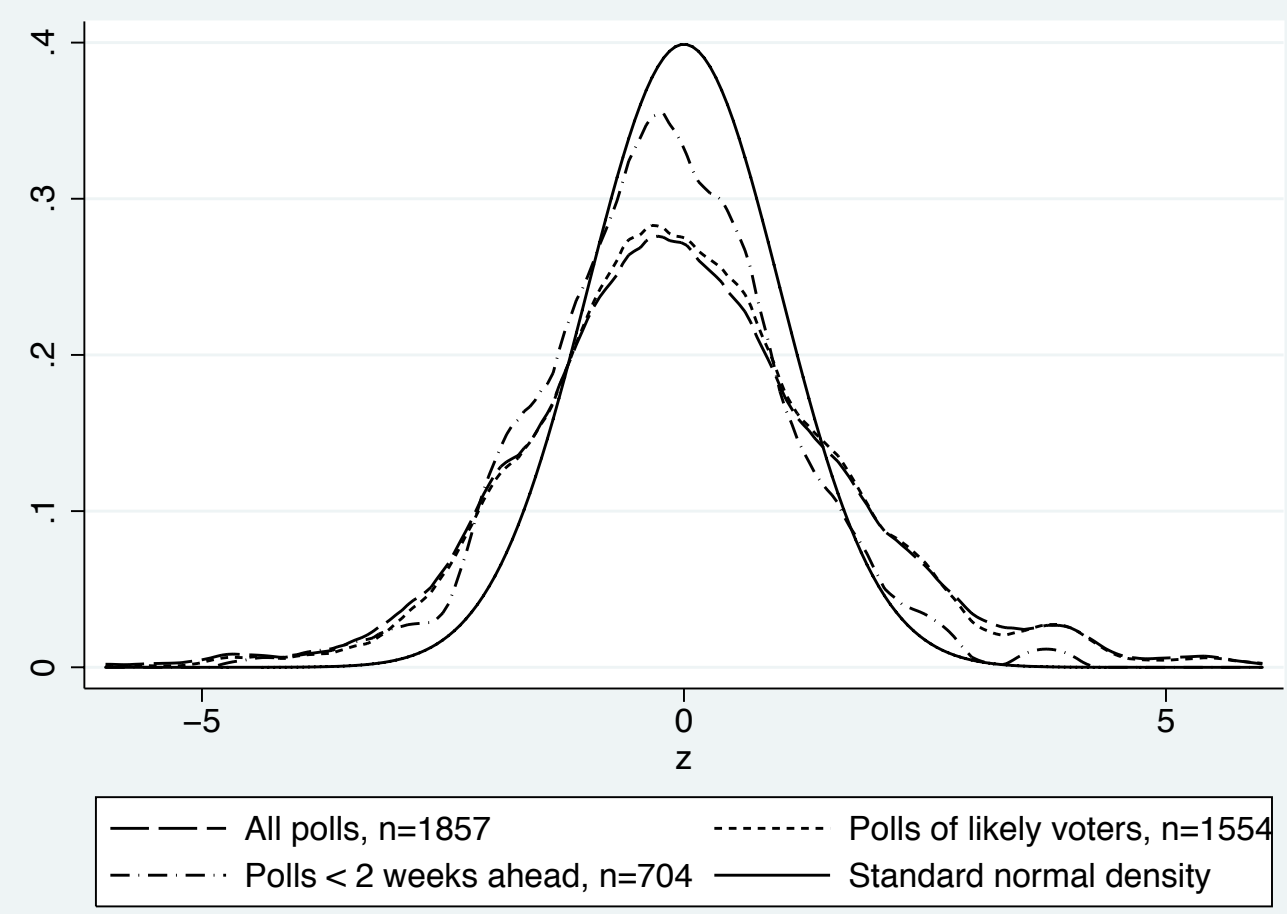

(a) Republican Prediction Error

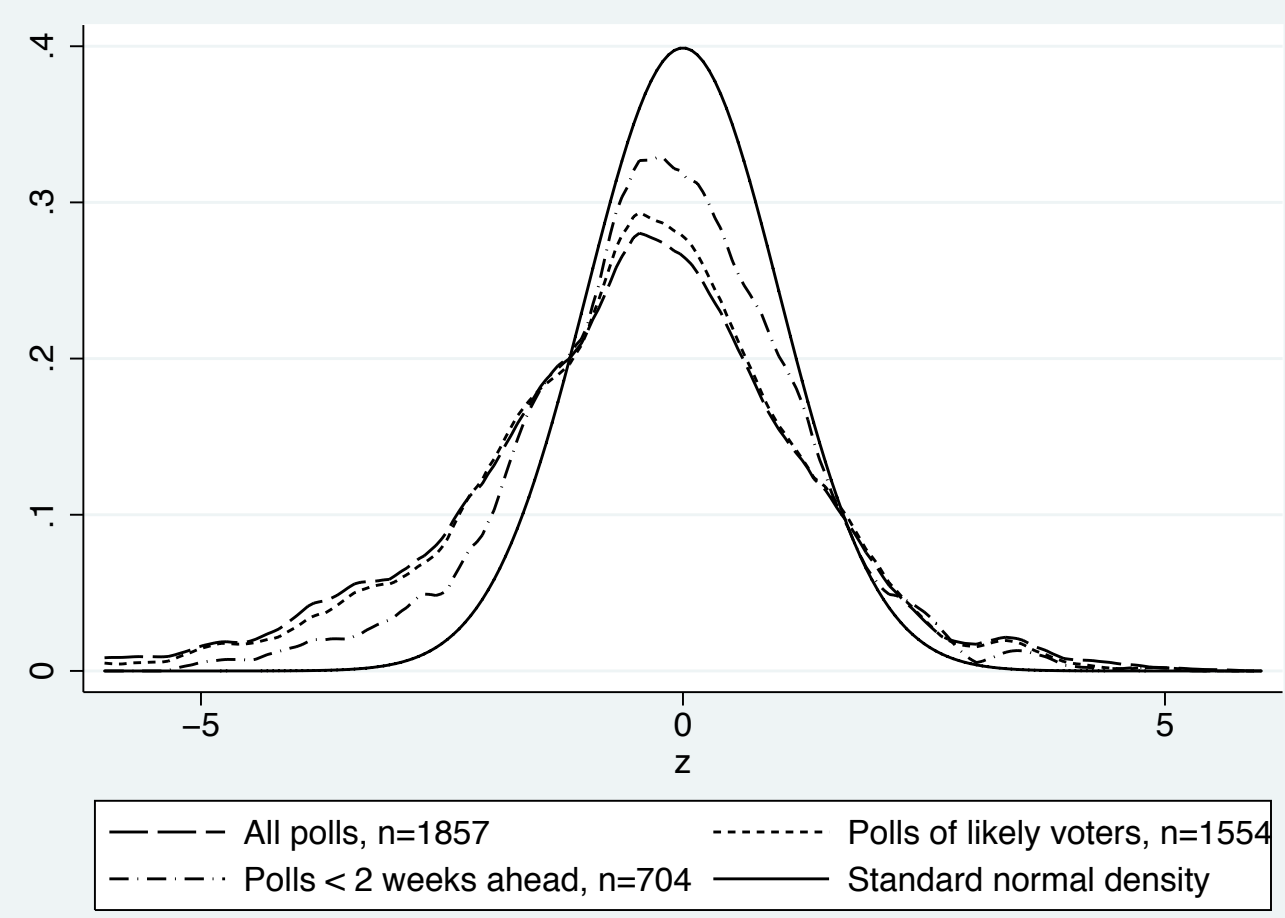

(b) Democratic Prediction Error

The figure displays a kernel density of the standardized prediction errors for presidential state races by poll subgroup. 
Web Appendix Figure 3: The Relation Between Forecasts and Election Results, Democratic Vote Share

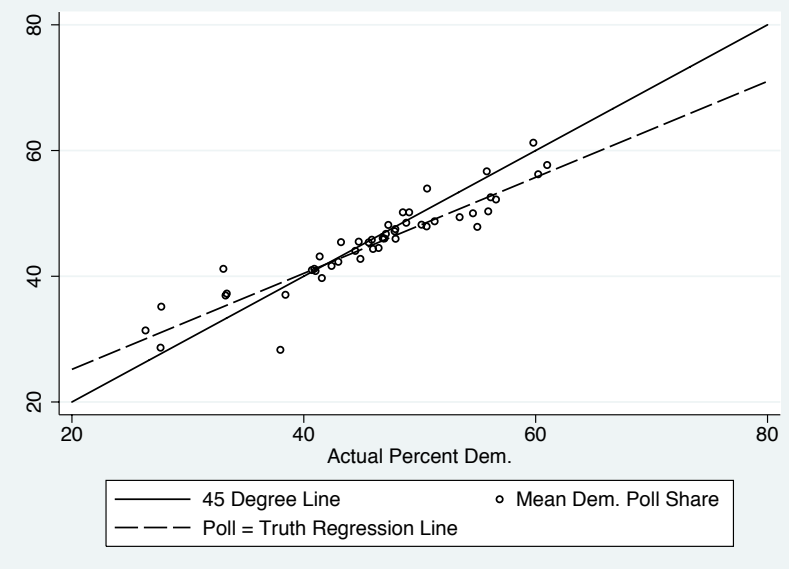

(a) All Polls, 2000

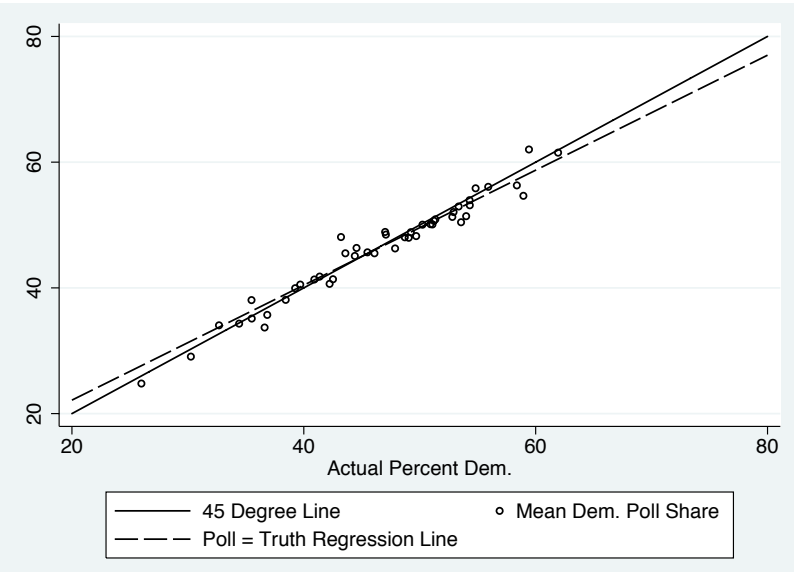

(c) All Polls, 2004

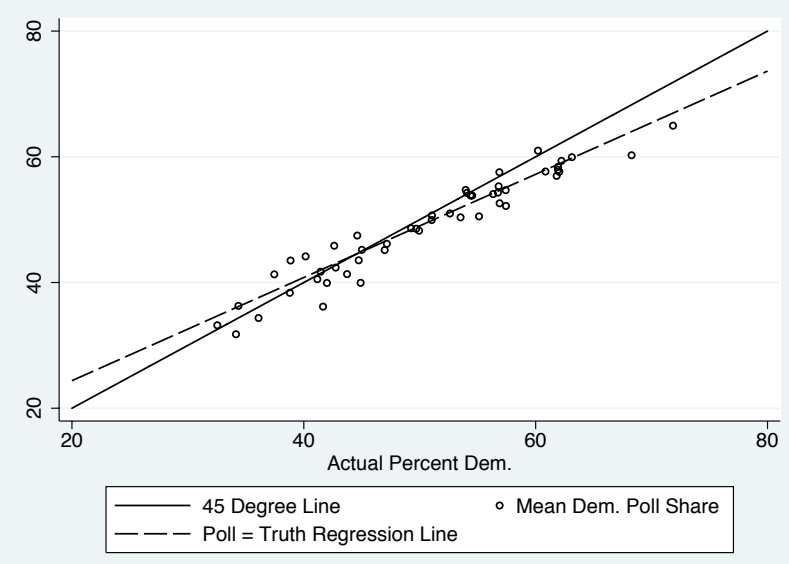

(e) All Polls, 2008

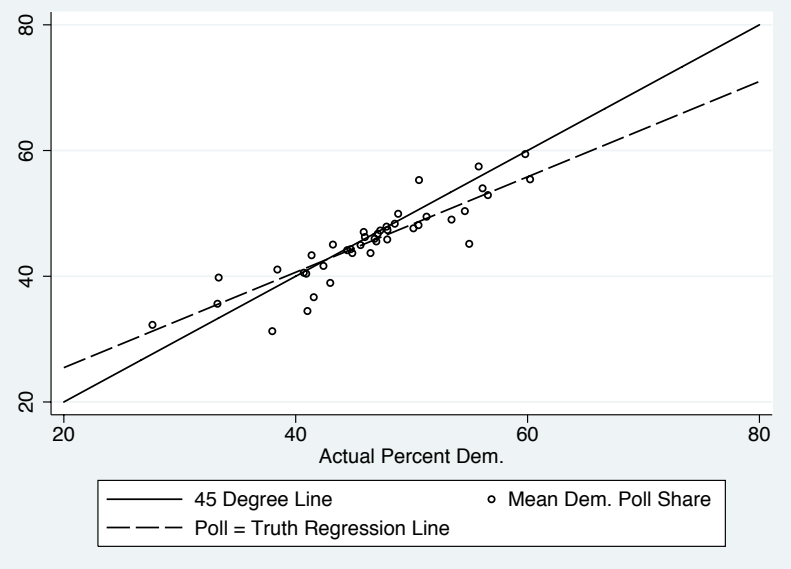

(b) Polls Within Two Weeks of Election, 2000

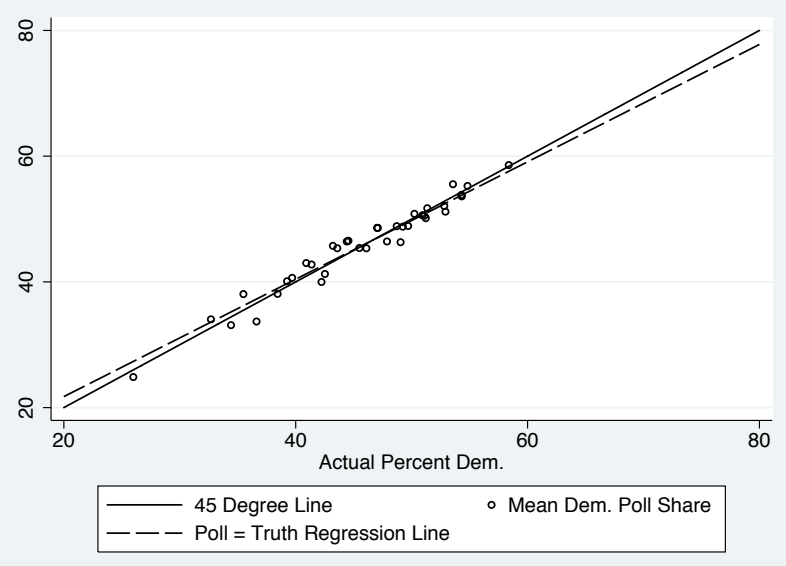

(d) Polls Within Two Weeks of Election, 2004

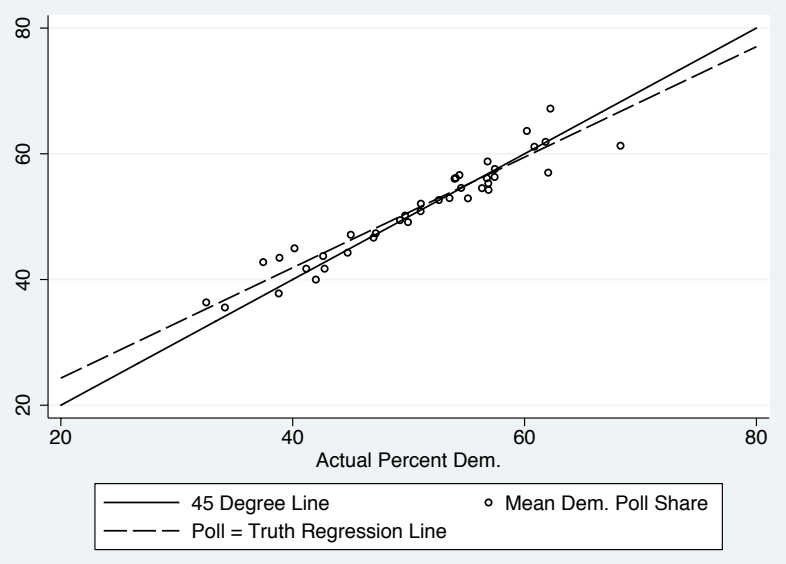

(f) Polls Within Two Weeks of Election, 2008

Each circle represents the mean of all poll results in a statewide election. The dashed line is the estimated line from a regression of the poll prediction on the actual election outcome. The solid line is the 45-degree line. 
Web Appendix Figure 4: The Relation Between Forecasts and Election Results, Republican Vote Share

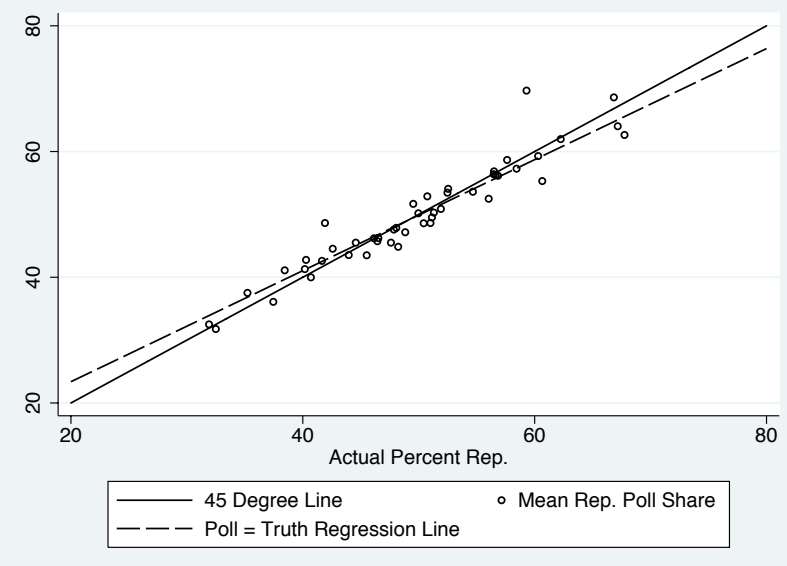

(a) All Polls, 2000

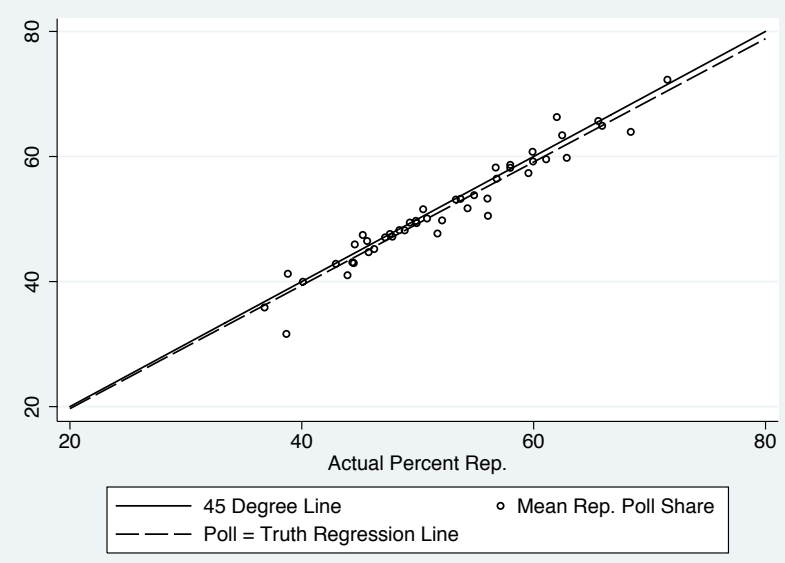

(c) All Polls, 2004

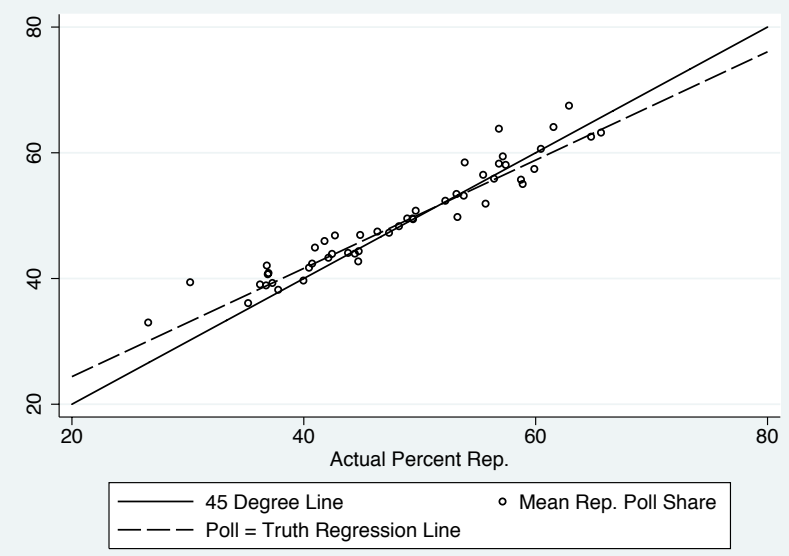

(e) All Polls, 2008

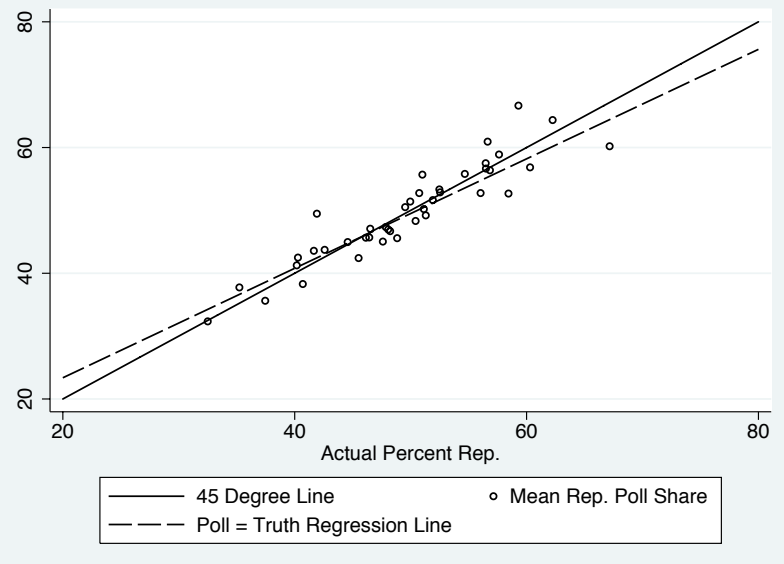

(b) Polls Within Two Weeks of Election, 2000

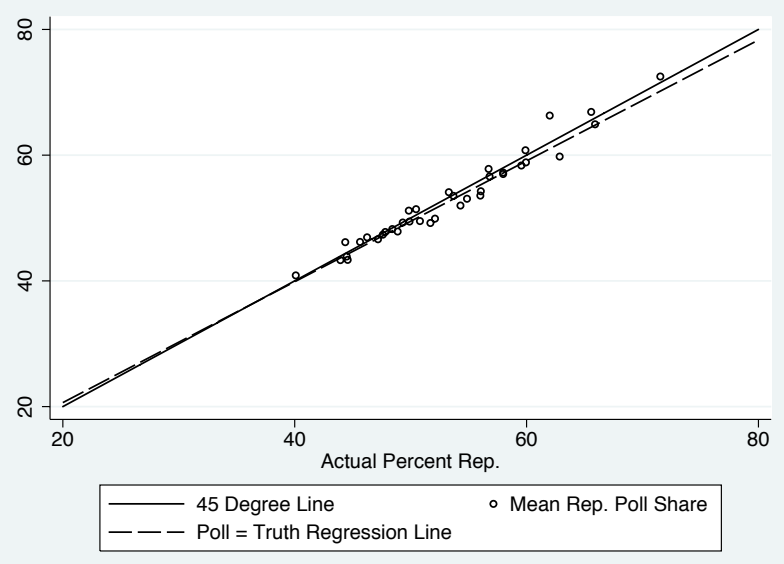

(d) Polls Within Two Weeks of Election, 2004

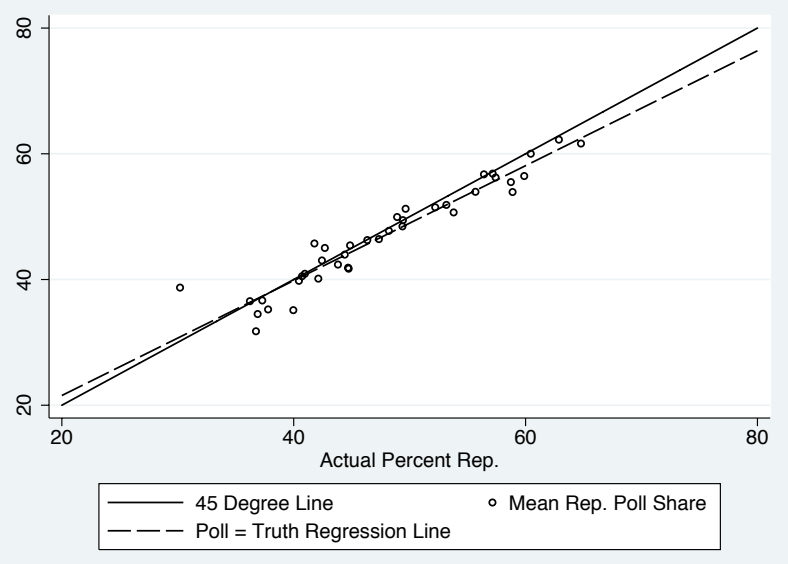

(f) Polls Within Two Weeks of Election, 2008

Each circle represents the mean of all poll results in a statewide election. The dashed line is the estimated line from a regression of the poll prediction on the actual election outcome. The solid line is the 45-degree line. 
Web Appendix Figure 5: The Relation Between Forecast Errors and Election Results, Democratic Vote Share

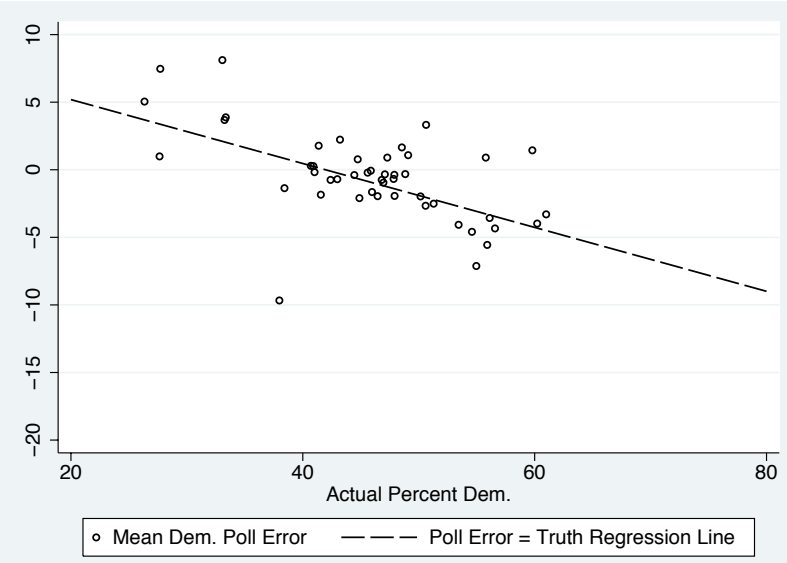

(a) All Polls, 2000

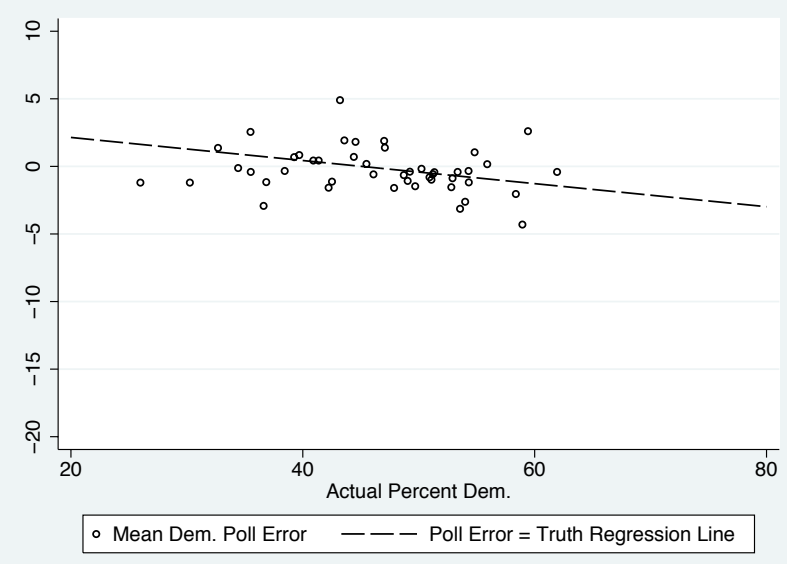

(c) All Polls, 2004

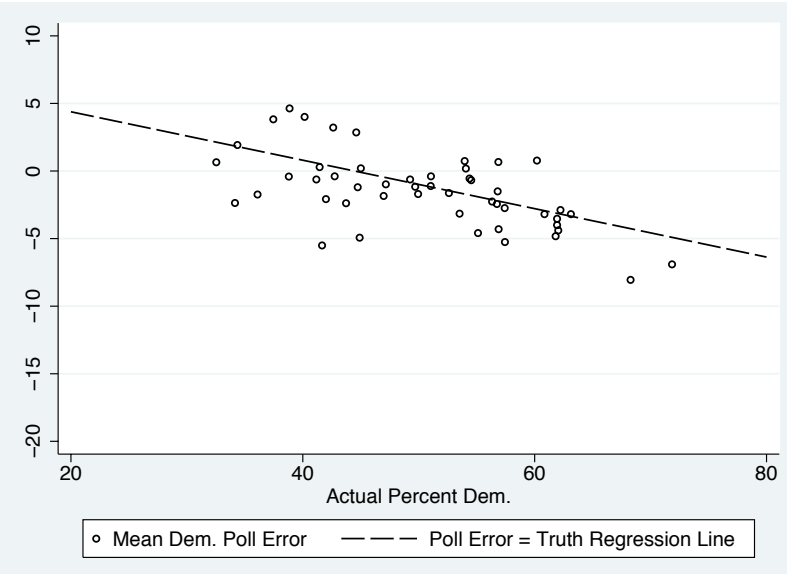

(e) All Polls, 2008

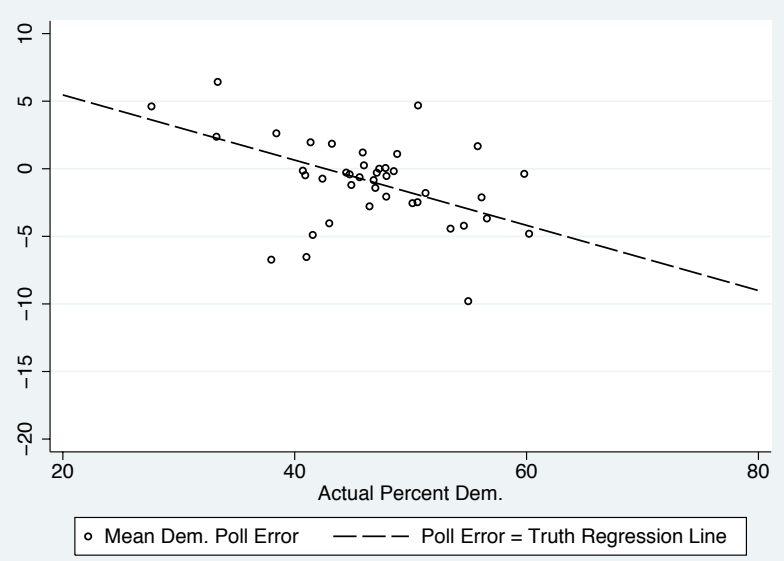

(b) Polls Within Two Weeks of Election, 2000

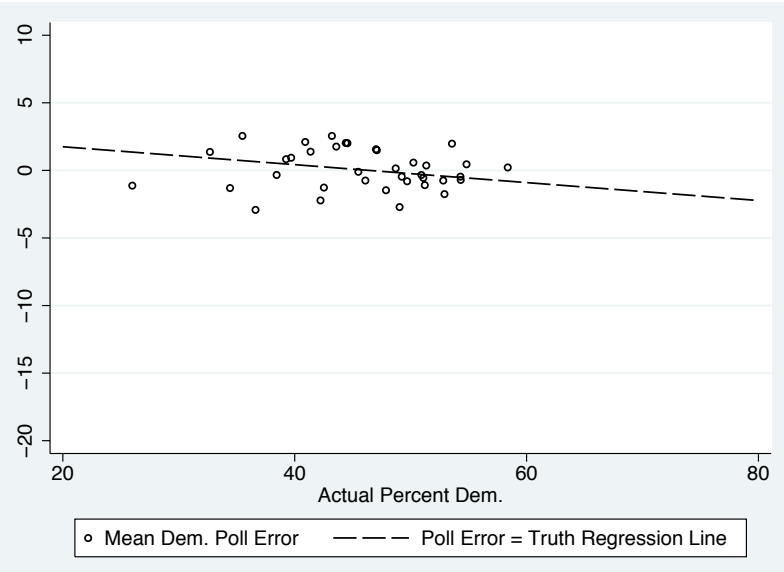

(d) Polls Within Two Weeks of Election, 2004

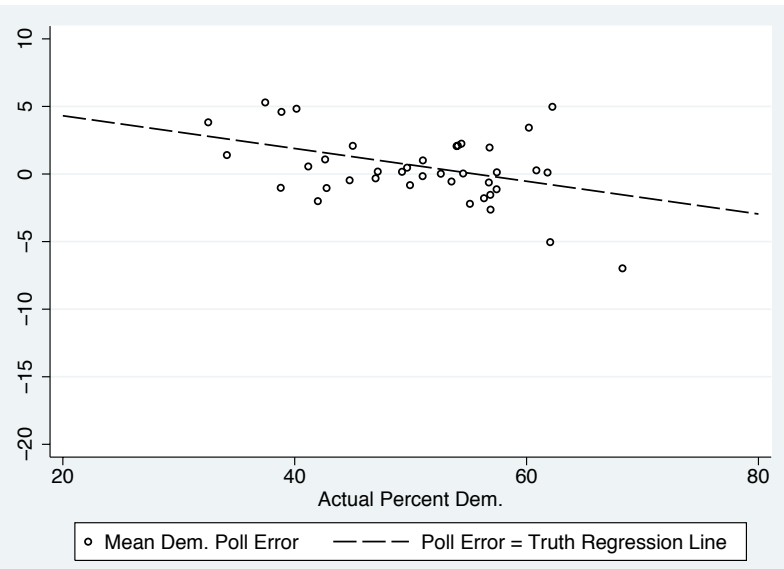

(f) Polls Within Two Weeks of Election, 2008

Each circle represents the mean of all poll prediction errors in a statewide election. The dashed line is the estimated line from a regression of the poll prediction errors on the actual election outcome. 
Web Appendix Figure 6: The Relation Between Forecast Errors and Election Results, Republican Vote Share

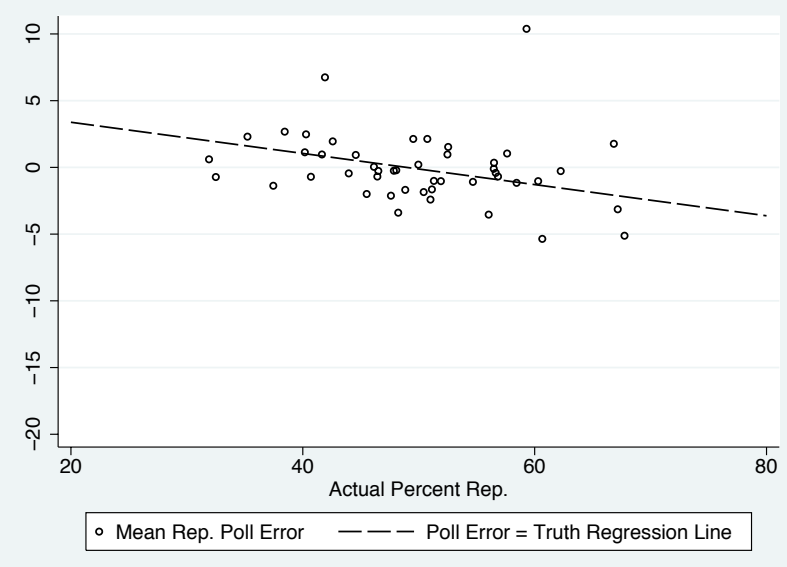

(a) All Polls, 2000

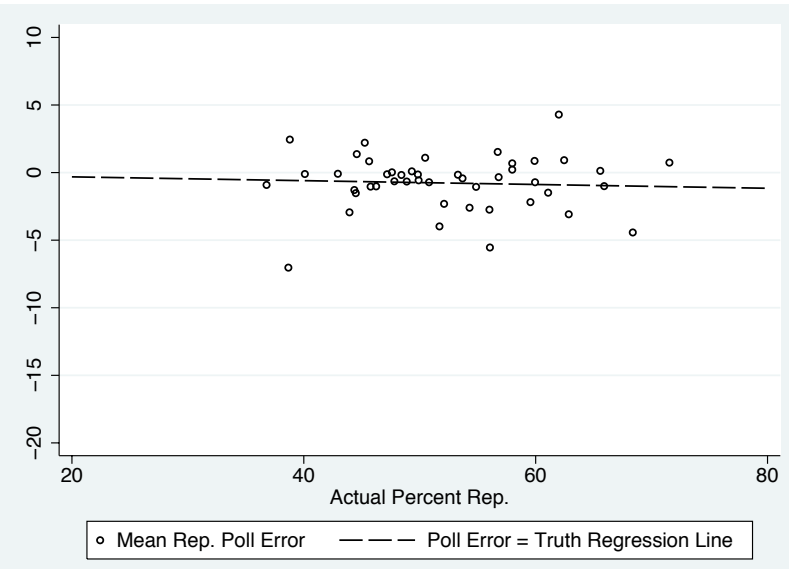

(c) All Polls, 2004

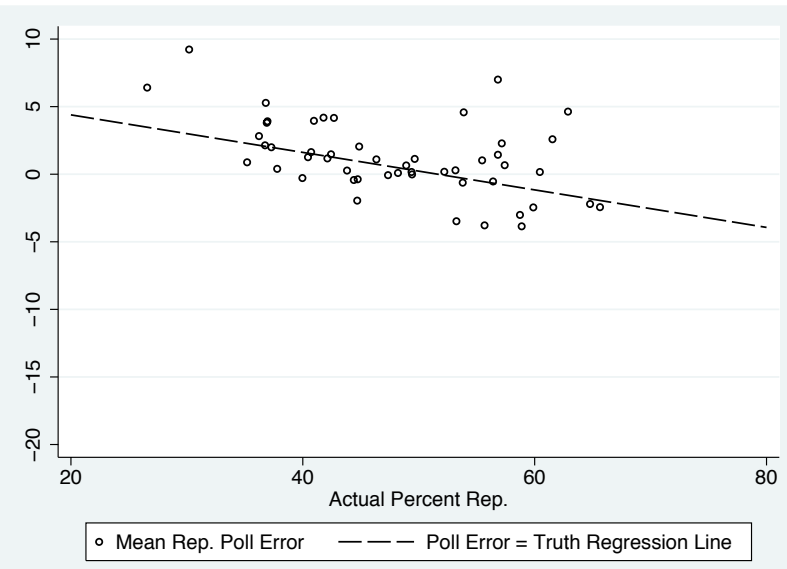

(e) All Polls, 2008

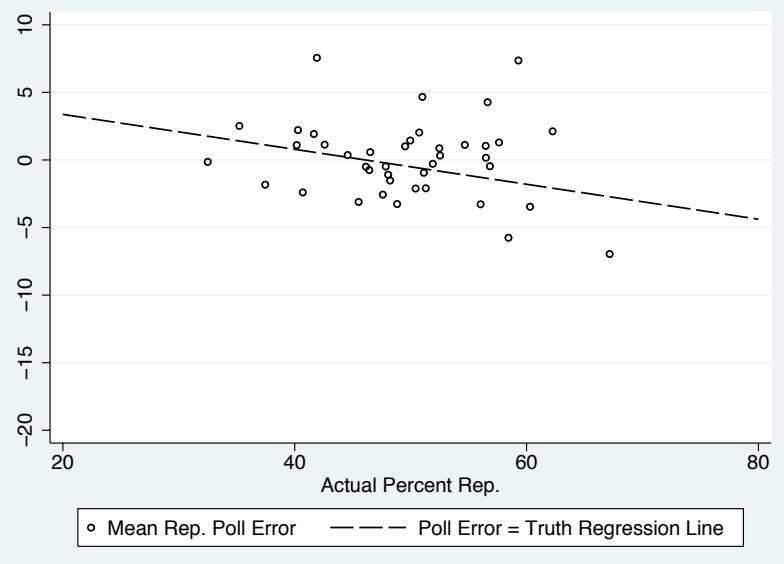

(b) Polls Within Two Weeks of Election, 2000

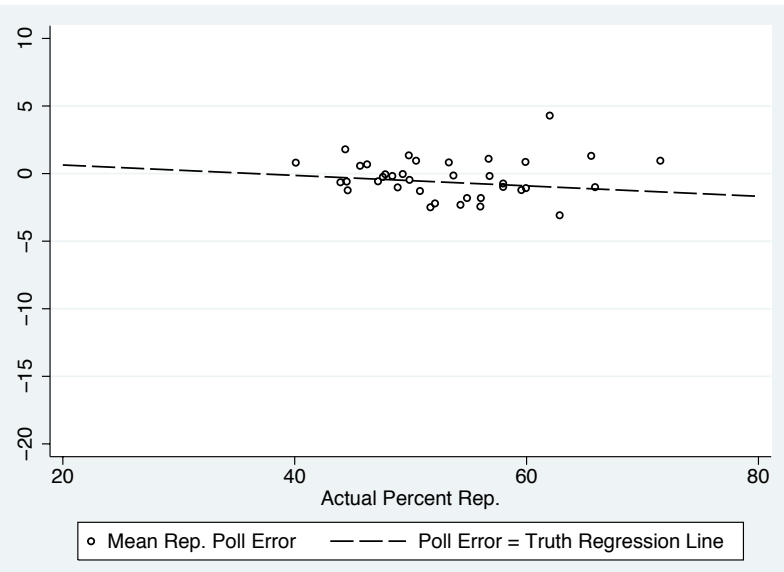

(d) Polls Within Two Weeks of Election, 2004

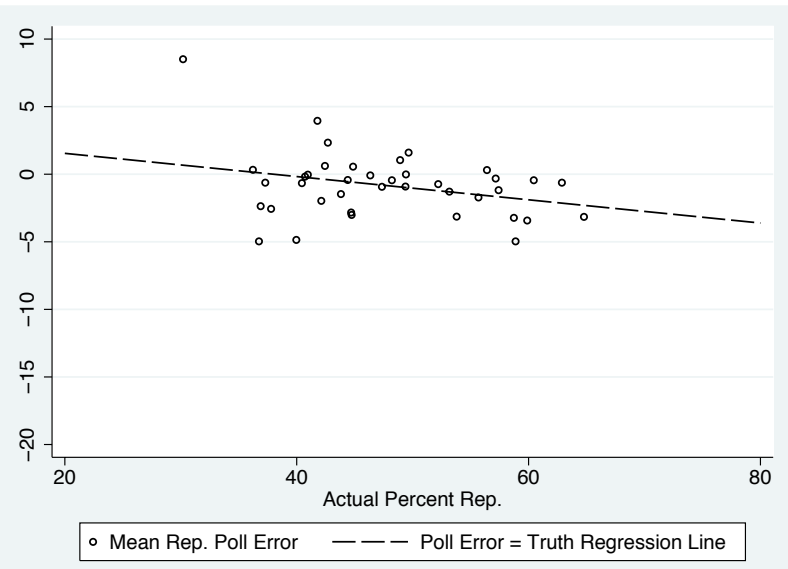

(f) Polls Within Two Weeks of Election, 2008

Each circle represents the mean of all poll prediction errors in a statewide election. The dashed line is the estimated line from a regression of the poll prediction errors on the actual election outcome. 
Web Appendix Figure 7: Distribution of Polls Across States By Election Result and Number of Electoral Votes

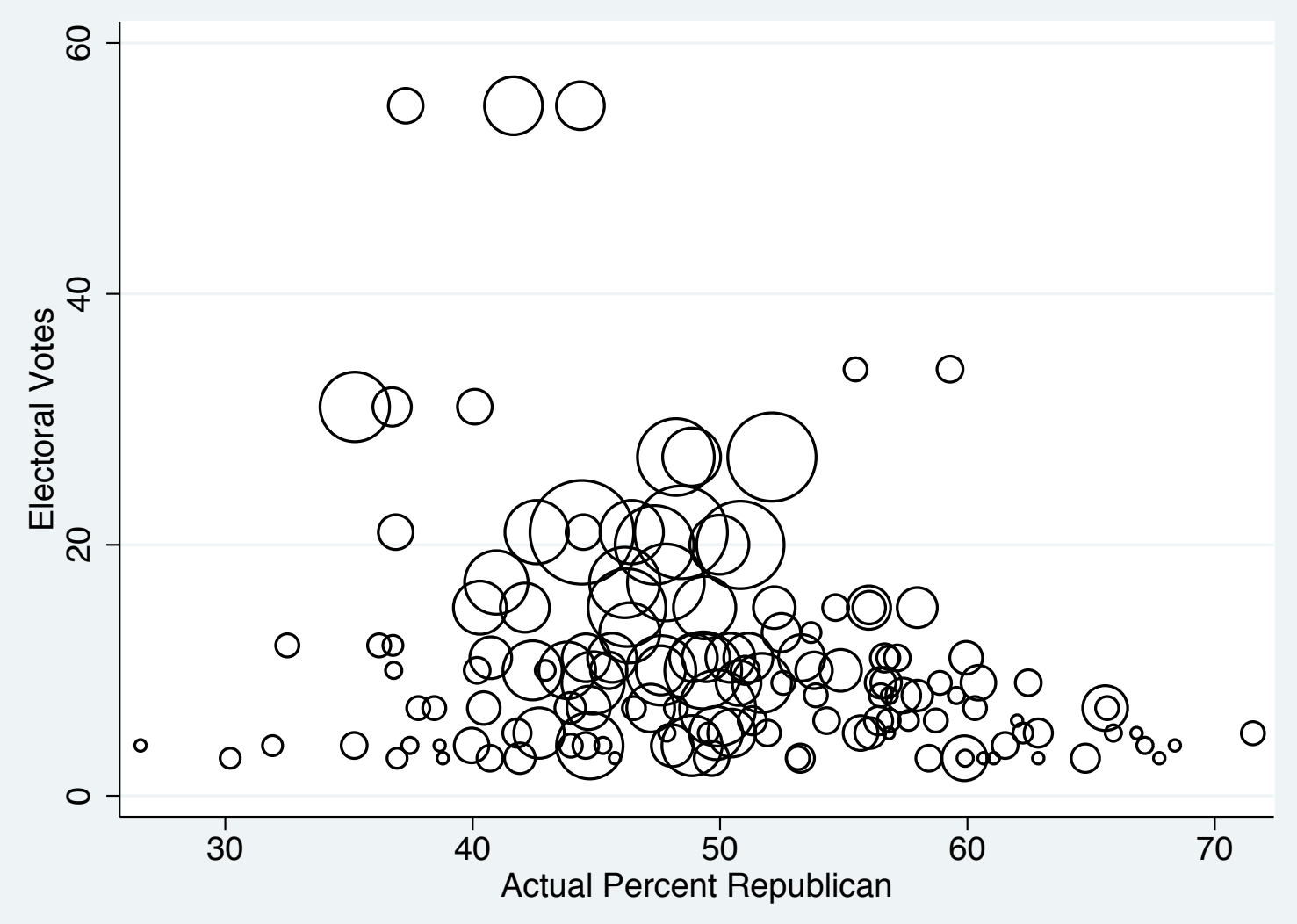

Each circle represents a statewide election. The area of the circle is proportional to the number of polls in that race. 
Web Appendix Figure 8: Standardized Prediction Errors Over Time

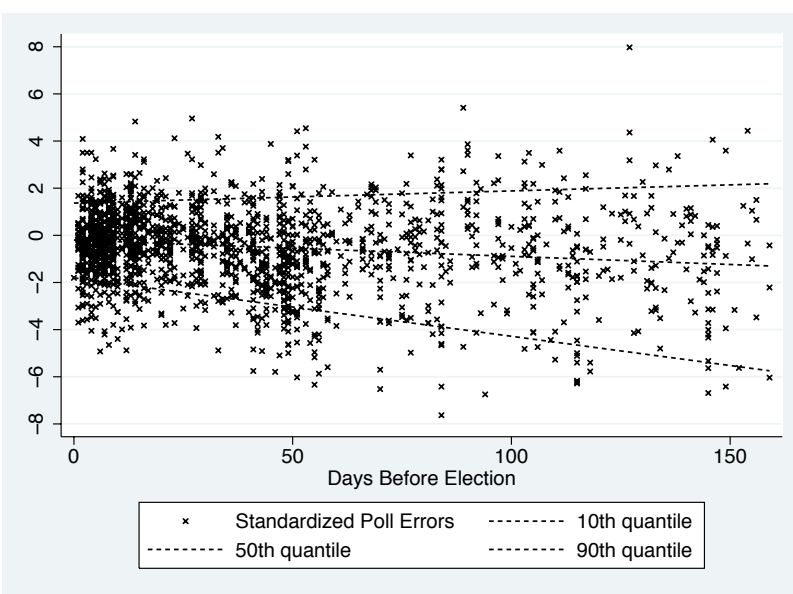

(a) All Democratic Standardized Prediction Errors

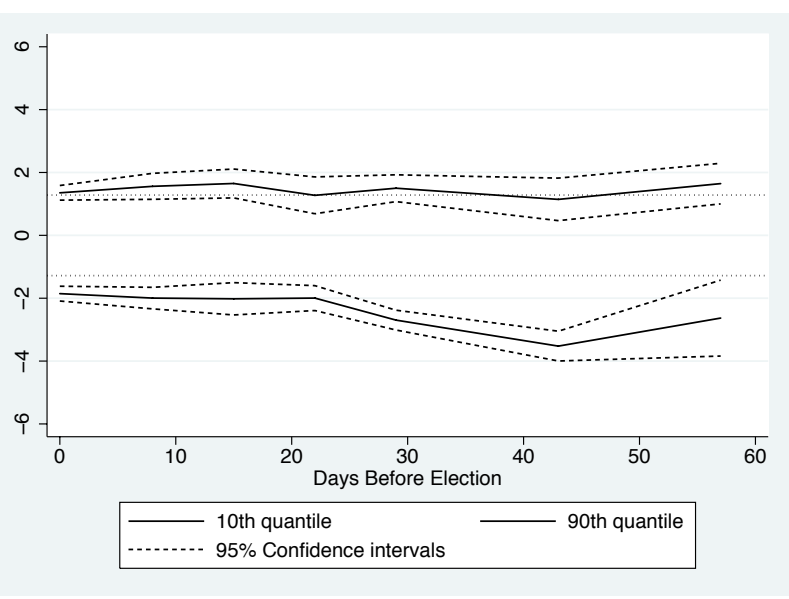

(b) All Democratic Standardized Prediction Errors

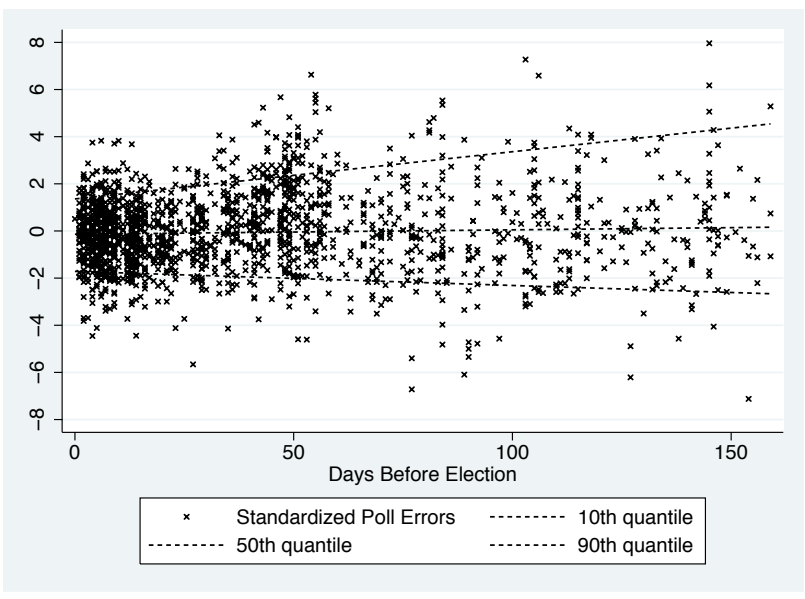

(c) All Republican Standardized Prediction Errors

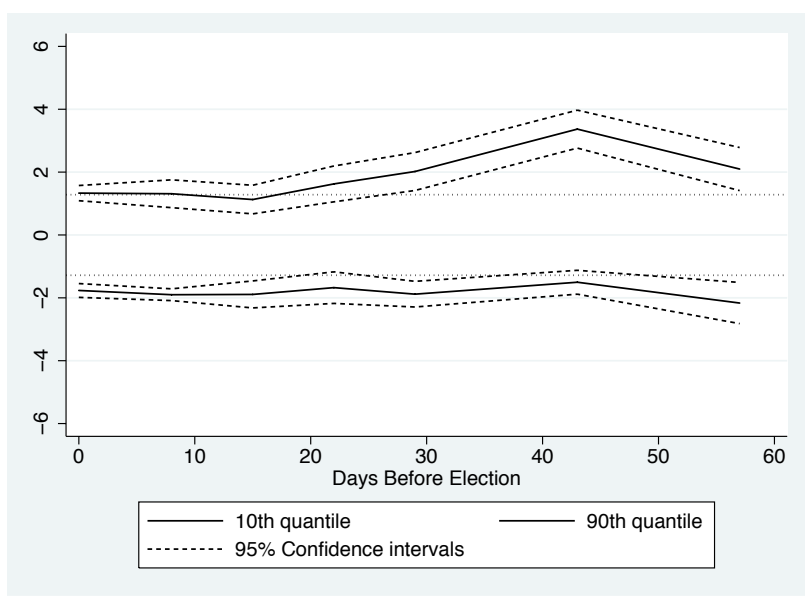

(d) All Republican Standardized Prediction Errors

The figure displays scatter plots of standardized prediction errors for presidential statewide races and quantile regressions at the 10th, 50th, and 90th quantile. The lines in panels (a) and (c) present the results of a quantile regression of the prediction errors on the number of days before the election and a constant term. Panels (b) and (d) present the 10th, and 90th quantiles and associated confidence intervals from a design-adaptive bandwidth quantile regression, limiting the sample to only those polls within 10 weeks of the election. The two dotted horizontal lines in each of panels (b) and (d) indicate the theoretical prediction of the 90th and 10th percentiles under standard normality $(1.28 /-1.28)$. 
Web Appendix Figure 9: Standardized Democratic Prediction Errors Over Time, by Election Year

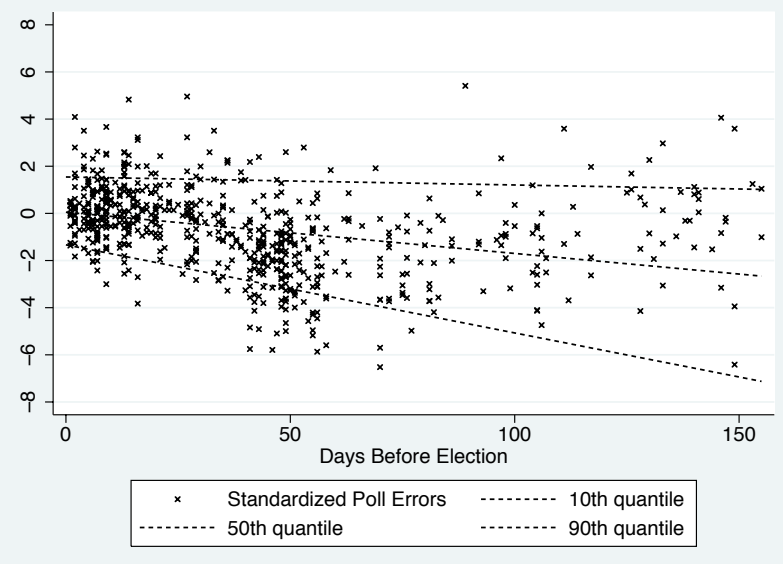

(a) 2008 Standardized Prediction Error

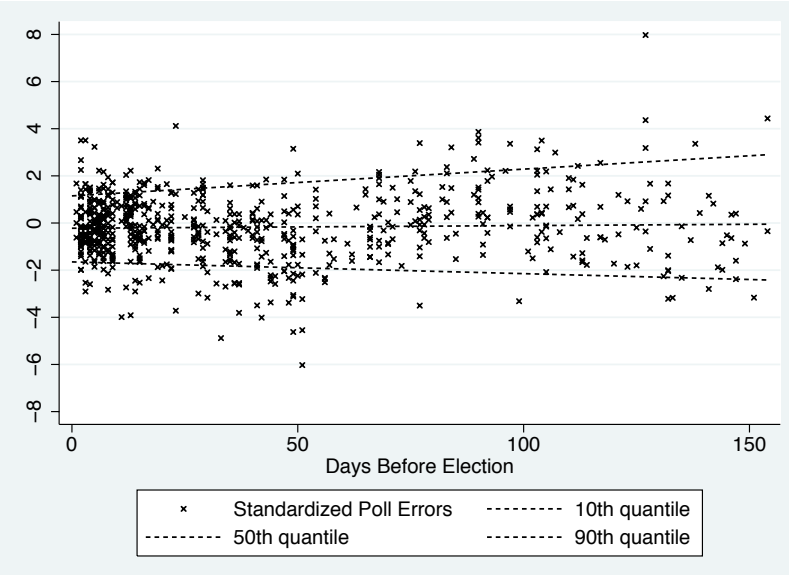

(c) 2004 Standardized Prediction Error

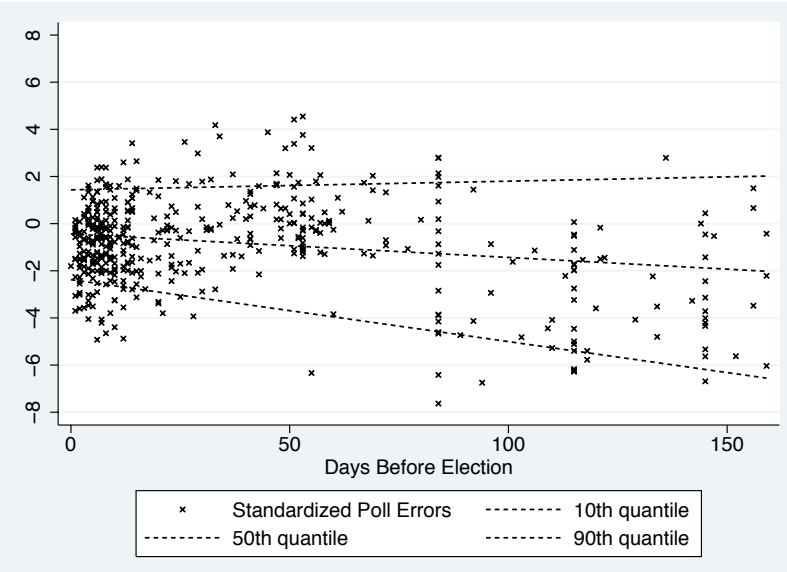

(e) 2000 Standardized Prediction Error

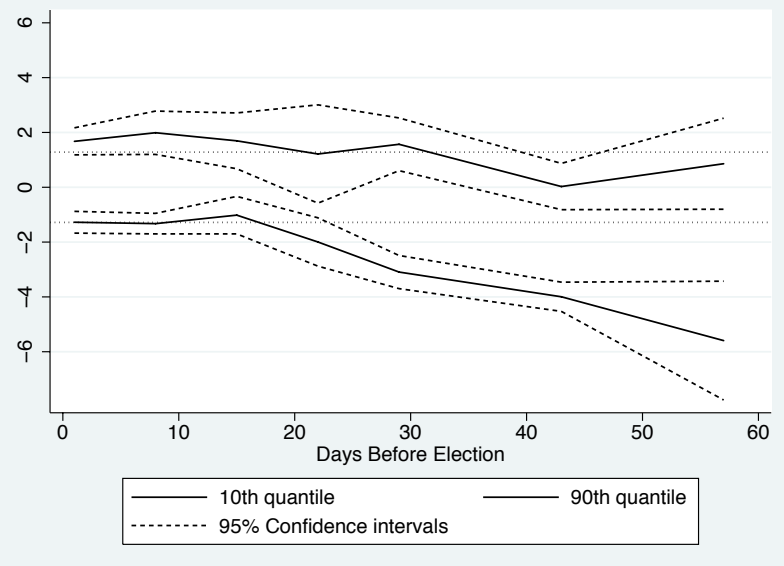

(b) 2008 Standardized Prediction Error

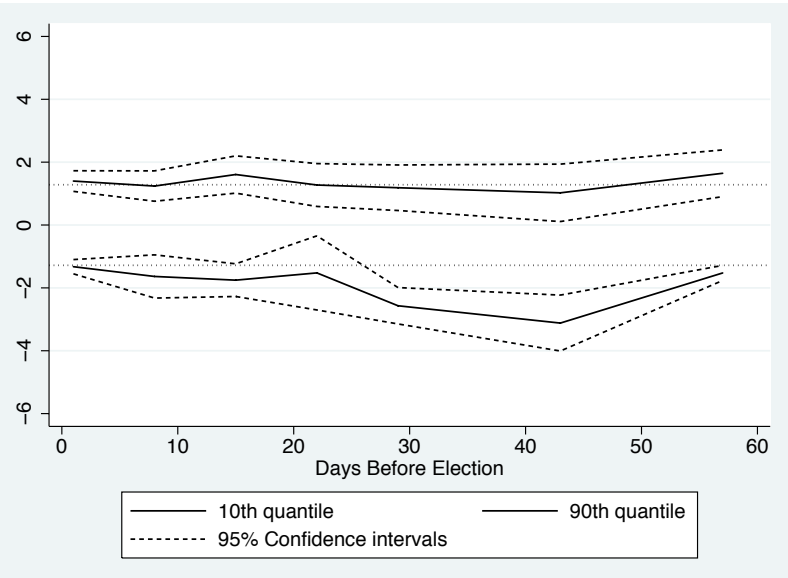

(d) 2004 Standardized Prediction Error

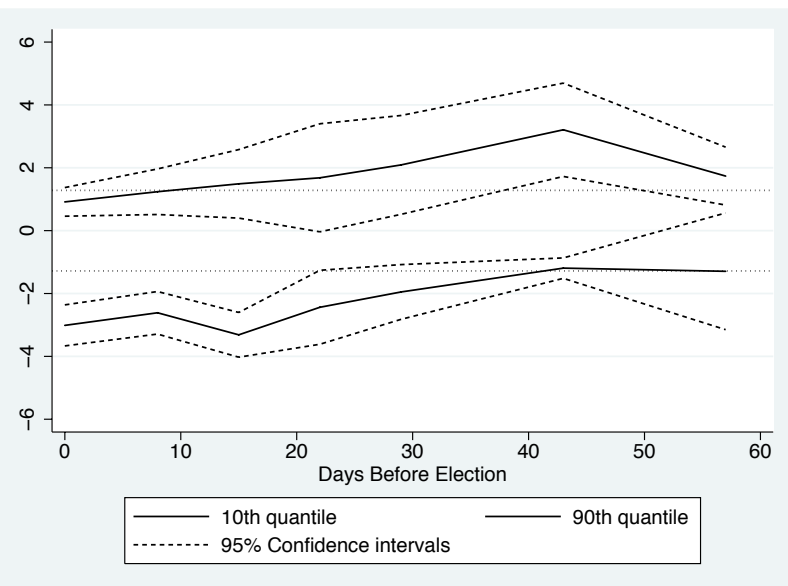

(f) 2000 Standardized Prediction Error

The figures display scatter plots of standardized prediction errors for presidential statewide races and quantile regressions at the 10th, 50th, and 90th quantile for polls separately by election year. The lines in panels (a), (c), and (e) present the results of a quantile regression of the prediction errors on the number of days before the election and a constant term. Panels (b), (d), and (f) present the 10th, and 90th quantiles and associated confidence intervals from a design-adaptive bandwidth quantile regression, limiting the sample to only those polls within 10 weeks of the election. The two dotted horizontal lines in each of panels (b), (d), and (f) indicate the theoretical prediction of the 90th and 10th percentiles under standard normality (1.28/-1.28). 
Web Appendix Figure 10: Standardized Republican Prediction Errors Over Time, by Election Year

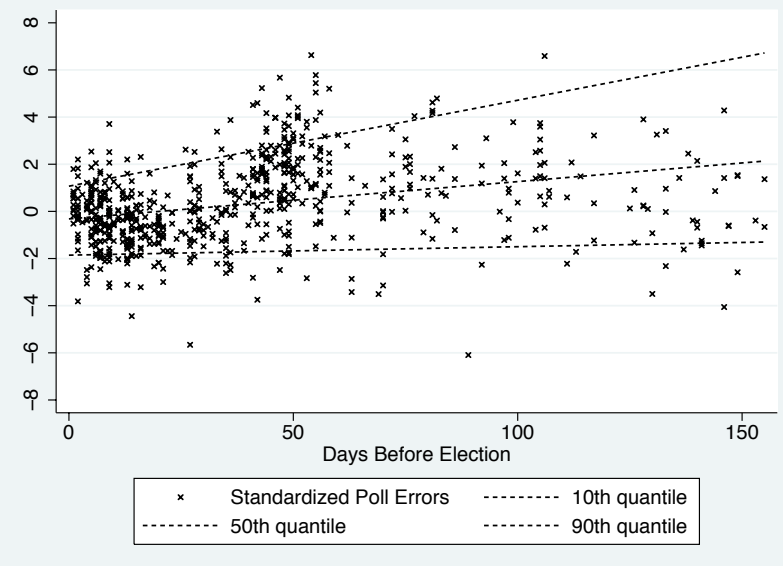

(a) 2008 Standardized Prediction Error

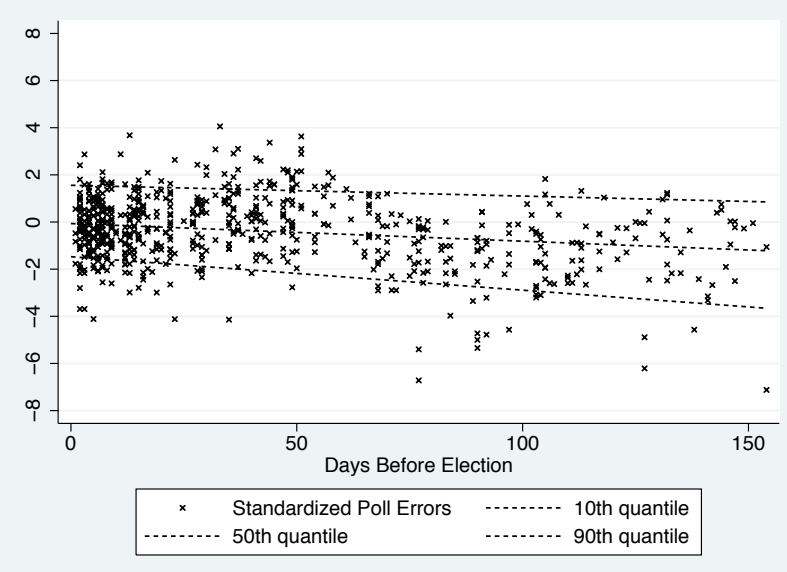

(c) 2004 Standardized Prediction Error

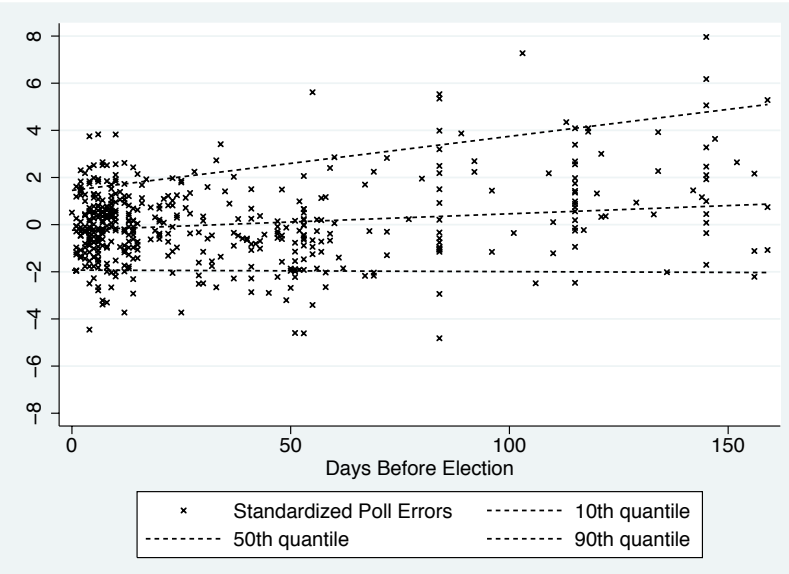

(e) 2000 Standardized Prediction Error

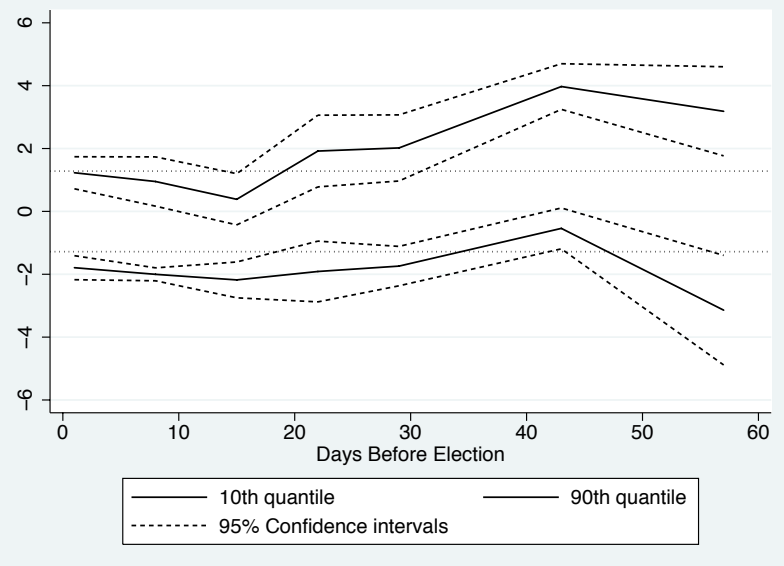

(b) 2008 Standardized Prediction Error

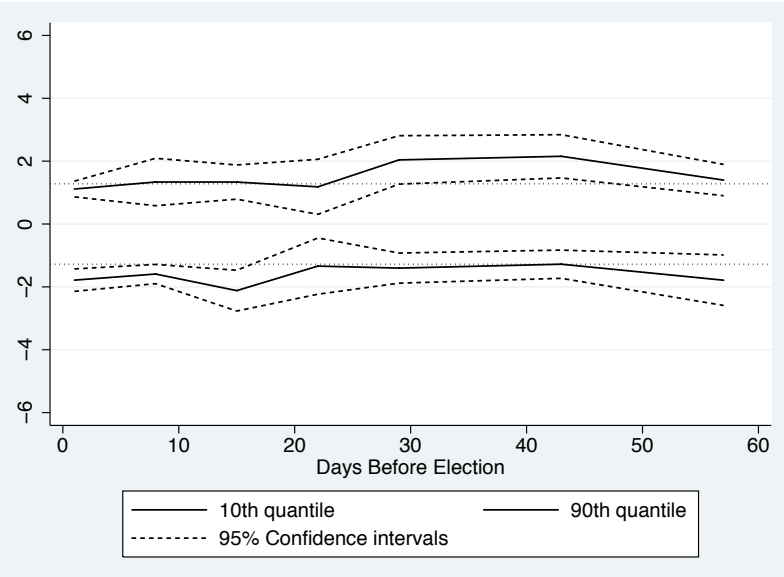

(d) 2004 Standardized Prediction Error

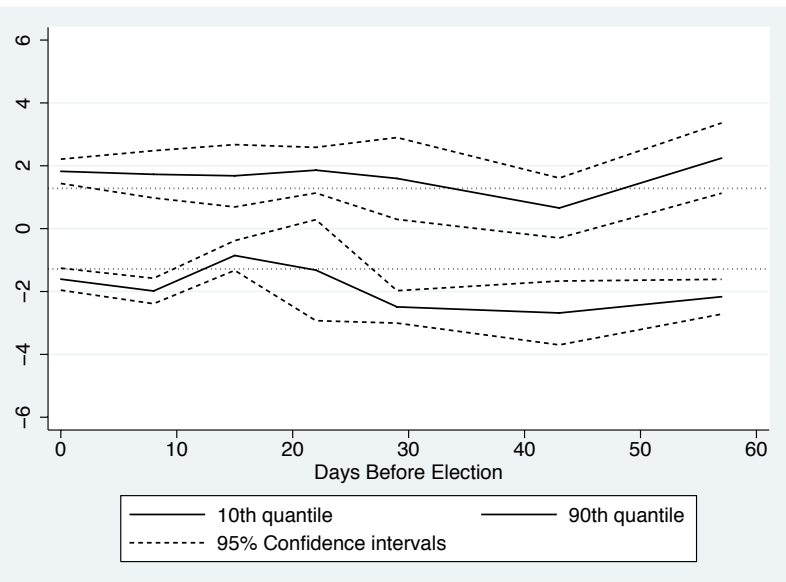

(f) 2000 Standardized Prediction Error

The figures display scatter plots of standardized prediction errors for presidential statewide races and quantile regressions at the 10th, 50th, and 90th quantile for polls separately by election year. The lines in panels (a), (c), and (e) present the results of a quantile regression of the prediction errors on the number of days before the election and a constant term. Panels (b), (d), and (f) present the 10th, and 90th quantiles and associated confidence intervals from a design-adaptive bandwidth quantile regression, limiting the sample to only those polls within 10 weeks of the election. The two dotted horizontal lines in each of panels (b), (d), and (f) indicate the theoretical prediction of the 90th and 10th percentiles under standard normality (1.28/-1.28). 
Web Appendix Figure 11: Density Estimates of Standardized Prediction Errors, by Detailed Poll Subgroup

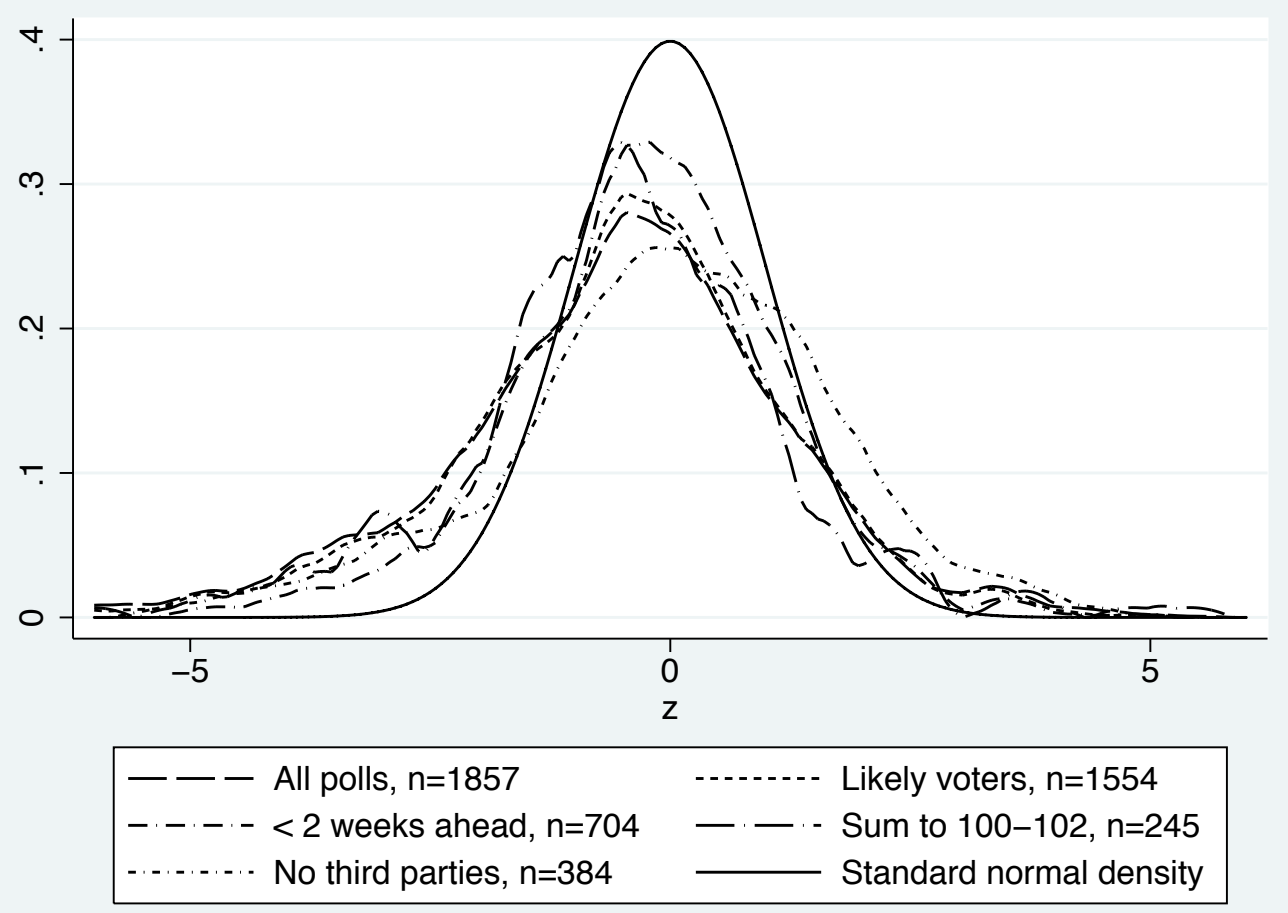

(a) Democratic Prediction Error

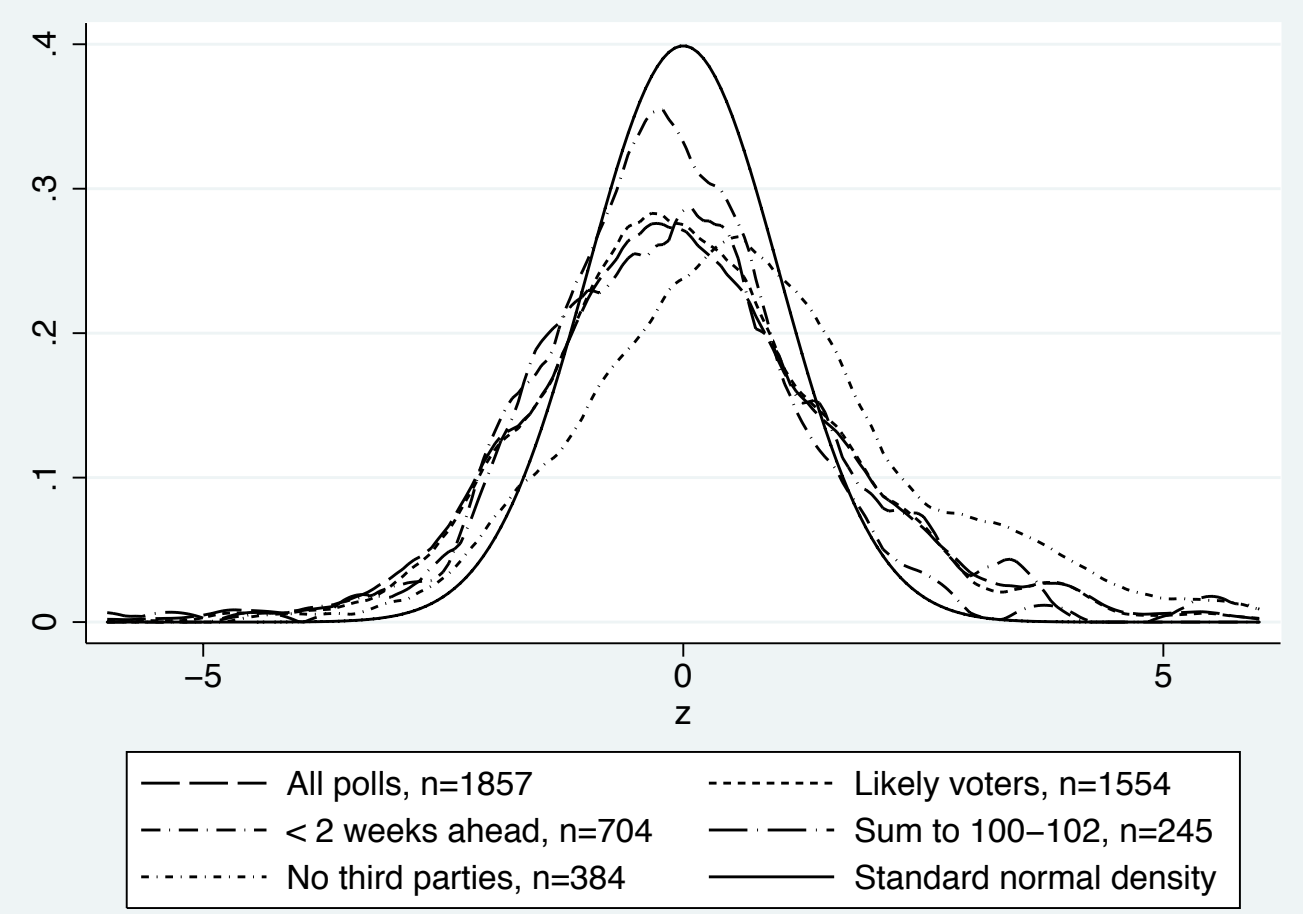

(b) Republican Prediction Error

The figure displays a kernel density of the standardized prediction errors for presidential state races by poll subgroup. 


\title{
Web Appendix: Discussion of Intentions and Polling
}

\author{
Elias Walsh, Sarah Dolfin and John DiNardo
}

January 30, 2009

\section{Probabilistic Intentions}

While a large literature (see Crespi (1988) for a nice summary) suggests that "horse race" polls those that ask respondents about who they intend to vote for in an election - should, if conducted properly and under the right conditions, reflect actual outcomes, an old statistical literature, most recently Manski (1990) suggests the opposite. Manski (1990) observes that if a potential voter is uncertain about who s/he will vote then a simple "intention" question: "who are you likely to vote for" will be biased in general for the outcome even if agents are perfectly rational, etc. The only hope for generating an unbiased prediction of an outcome from intentions data requires asking the question in such a way that allows the voter to express his or her uncertainty.

Instead of asking: If the election were held today, would you:

- Vote for John Kerry, the Democratic nominee for president.

- Vote for George Bush, the Republican nominee for president.

- Vote for another candidate.

one should ask the question in terms of probabilities for voting for each of the candidates.

It seems worthwhile to ask whether this "theoretical" source of bias can explain much of the bias we observe in actual polls. In a sense, we would like to see the extent to which this purely "statistical" problem addresses the question posed by Gelman and King (1993) - are polls variable only because the questions are posed as intentions instead of probabilities? The purpose of this section of the paper is to investigate the importance of this question by a comparison of responses to "horse race" questions asked the usual way, and the way suggested by Manski's analysis. Both trends and the reliability of the implied forecast may be quite different for the two sets of questions and this might yield insights as to why polls tend to be biased forecasts of the outcomes.

While this source of bias has been studied extensively for continuous outcomes such as income (see Dominintz and Manski (1997) for a review and example) to the best of our knowledge has not been studied in this context. This problem arises routinely in data of interest to political scientists, economists, sociologists and others and may have implications for broader issues than merely horse race election polling per se.

Although "horse race" polls are routinely used to forecast the likelihood that some candidate will win an election, it is well understood in the statistics literature that even in the "best case" there is no reason to suppose that "intentions" ("I am likely to vote for candidate X") should yield unbiased forecasts of actual behavior. (Manski, 1990) 
We first focus on a "best case" scenario and illustrate with some simple numerical examples why

1. Polls should be biased in general.

2. Even large positive changes in poll results over time do not necessarily indicate increased support for the candidate.

In doing so, we focus only on the possibility that some individuals are uncertain about who they will vote for. We assume that all the other possible problems (sample selection biases, question ordering, etc.) that have been cited in the literature are solved. ${ }^{1}$ As a rule, assuming something worse than the "best case" results in an even greater bias and for reasons of brevity and clarity we omit that discussion here.

\section{A The Best Case}

Following Manski (1990), let $i$ be a binary indicator denoting an intention - "talking about the presidential elections in November, for whom are you likely to vote - George Bush?" and let $y$ be the indicator corresponding to the actual behavior (the individual votes for Bush). Letting $s$ denote the information available at the time of the survey to the respondent and let $z$ denote the events that have not yet occurred but that will affect his future action. ${ }^{2}$ Let $P_{z} \mid s$ denote the objective distribution of $z$ conditional on $s$. Let $P(y \mid s)$ denote the objective distribution of $y$ conditional on $s$. The event $y=1$ occurs $\Longleftrightarrow$ the realization of $z$ is such that $y(s, z)=1$.

In the best case, we assume rational expectations: this means the respondent knows how they will act depending on the possible realizations of $z$ and that they also know $P_{z} \mid s$ - that is they know the stochastic process generating $z$-in words, the respondent knows the correct distribution of the behavior influencing events $z$ and moreover uses that information optimally. To take a concrete example, suppose $z$ is the public exposure of a scandal involving "morals" or sexual behavior of a candidate. This assumption is the requirement that I know how I would behave if my candidate were involved in a scandal and the the probability that I would learn about such a scandal before election day.

The second aspect of the "best case" scenario is that the respondent states her best point prediction of her behavior. The best prediction depends on her "loss function" associated with either $(i=1, y=0)$ and $(i=0, y=1)$. Manski observes that under these two sets of assumptions the responses satisfy:

$$
\begin{aligned}
& i=1 \quad \Longrightarrow \quad P(y=1 \mid s) \geq \pi \\
& i=0 \quad \Longrightarrow \quad P(y=1 \mid s) \leq \pi
\end{aligned}
$$

In words, if the action $y$ is "voting for candidate X", then a respondent tells the interviewer that she will vote for candidate $\mathrm{X}$ if the probability that she will do so is greater than $\pi$. If both possible errors are equally "costly" than $\pi=.5$ Specializing to the case of horse race polls, the object of the poll is to learn the probability $P(y=1 \mid i, s)$. As Manski observes, however, the pollster's data on "intentions" does not identify that probability. Even in this "best case" - assuming that persons have identical loss functions - they only imply a "bound". As Manski shows:

\footnotetext{
${ }^{1}$ See for example, Gelman and King (1993) or Ottaviani and Norman (2006) for discussions.

${ }^{2}$ To make the problem even more simple, we assume that a person's participation is known with certainty. Allowing for uncertainty in participation only strengthens the negative result.
} 
Figure 1: The Bounds Implied by "Intentions" are not tight: A comparison of intentions with outcomes

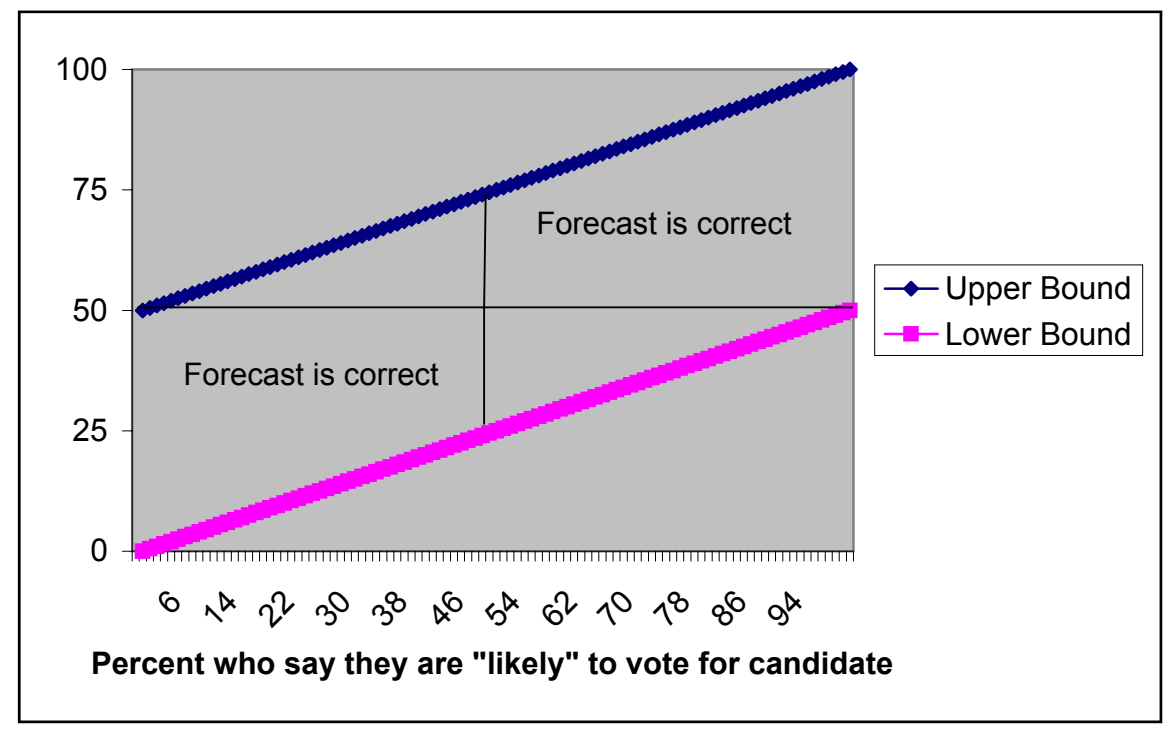

$$
P(y=1 \mid s, i=0) \leq \pi \leq P(y=1 \mid s, i=1)
$$

expresses all the information in intentions data.

Figure 1 displays the bounds implied by the data assuming no sampling error, that individuals have identical symmetric loss functions, and that there is no "new information" $s$ between the time the poll is taken and the behavior occurs. The dependent variable is the actual voting outcome on election day.

The lower right and upper left triangles that lie within the polygon formed by the bounds indicate that 25 percent of the area within the bounds fail even to cover the correct binary prediction of the outcome. Note that it would be incorrect to draw the inference that the polls would get it right 75 percent of the time in this best case. Rather, the correct inference is that the correct bounds do not have to cover the correct binary prediction of the election outcome. Of course, if the sample is not a random sample, new information occurs between the poll and the event, or that there is a double uncertainty (i.e. the voter does not know for certain whether s/he votes) the bounds could easily be completely uninformative.

\section{B A Rise in the Polls Doesn't Necessarily Imply Increased Support}

Observe that we have gone a bit beyond even the "best case" in this simple illustration. As Manski observes (and as was observed earlier by Juster (1966), for example) it has been well known in the statistical literature that such polls will not be unbiased in general, even in this best case. As a consequence, a poll is especially unsuited to assessing "trends" in voter support for a candidate, even when the electorate is composed of Bayesian statisticians with correct rational expectations. 
The following contrived example, although not altogether unreasonable, shows an example where support for candidate $\mathrm{X}$ is falling (measured as what would actually have happened if an election had been conducted), at the same time the polls are showing a massive increase in support for the candidate. For simplicity, we have three types of voters. Type "C" voters strongly support candidate X, type "B" voters less strongly support candidate X, and type "A" voters strongly oppose candidate X. Between the two periods, type "A" voters grow much more strongly opposed to candidate X, and type "B" voters slightly shift in favor of candidate X. As a consequence, the polling shows a large increase in support for candidate $\mathrm{X}$ from period 1 to period 2, even as the actual probability of $\mathrm{X}$ being elected fell over this time!

Table 1: Polls show increased support, when support is falling

\begin{tabular}{ccccccc}
\hline \hline & & & \multicolumn{2}{c}{ Time Period 1 } & & \multicolumn{2}{c}{ Time Period 2} \\
\cline { 3 - 4 } \cline { 6 - 7 } $\begin{array}{c}\text { Voter } \\
\text { "Type" }\end{array}$ & $\begin{array}{c}\text { Fraction } \\
\text { in Population }\end{array}$ & $\begin{array}{c}\text { Probability } \\
\text { vote for X }\end{array}$ & $\begin{array}{c}\text { Response } \\
\text { to Pollster }\end{array}$ & & $\begin{array}{c}\text { Probability } \\
\text { vote for X }\end{array}$ & $\begin{array}{c}\text { Response } \\
\text { to Pollster }\end{array}$ \\
\hline A & 0.25 & 0.4 & 0 & & .1 & 0 \\
B & 0.5 & 0.46 & 0 & & .51 & 1 \\
C & 0.25 & 0.8 & 1 & & Actual Outcome & 0.48 \\
\hline \hline & & Actual Outcome & 0.53 & & Poll Result & 0.75
\end{tabular}

\section{Voter Participation}

The above analysis applies mutatis mutandis to an analysis of voter participation as a separate inquiry. As far as we have been able to ascertain, polling organizations routinely use a binary measure of whether or not an individual is likely to vote. Again, if the decision to participate is uncertain, in general there is no reason to believe that restricting to the sample to "likely voters" or "registered voters" (the two most frequently used screens in practice) will yield an unbiased rate of participation.

Moreover, since - in the simplest model - the act of the voting for a specific candidate is the product of two uncertain decisions (a decision to support the candidate, and the act of going to the polling booth) it is clear that treating the corresponding sets of intentions as certain - i.e. binary - is biased as a forecast of the actual vote or the "strength" of the support for a candidate. 


\section{References}

Crespi, Irving, Pre-Election Polling: Sources of Accuracy and Error, New York: Russell Sage Foundation, 1988.

Dominintz, Jeff and Charles F. Manski, "Using Expectations Data to Study Subjective Income Expectations," Journal of the American Statistical Association, September 1997, 92 (439), 855867.

Gelman, Andrew and Gary King, "Why are American Presidential Election Campaign Polls So Variable When Votes are So Predictable?," British Journal of Political Science, October 1993, 23 (4), 409-451.

Juster, Thomas, "Consumer Buying Intentions and Purchase Probability: An Experiment in Survey Design," Journal of the American Statistical Association, 1966, 61, 658-696.

Manski, Charles F., "The Use of Intentions Data to Predict Behavior: A Best-Case Analysis," Journal of the American Statistical Association, December 1990, 85 (412), 934-940.

Ottaviani, Marco and Peter Norman, "The Strategy of Professional Forecasting," Journal of Financial Economics, August 2006, 81 (2), 441-446. 


\title{
Web Appendix: Poll Questions
}

\author{
Elias Walsh, Sarah Dolfin and John DiNardo
}

January 30, 2009

Our study design consisted of the following two pairs of questions:

For the first demographically balanced half-sample, the questions are designed to allow respondents to express probabilistic intentions:

1. Are you a registered voter? If yes:

- Given your other obligations, on a scale of 0 to 100 what is the chance that you will actually cast a vote for president? If you are certain you will vote, state "100." If you are certain you will not vote, state " 0 ". If there is a 40 in 100 chance you will vote, state 40, and so on.

If no,

- Given your other obligations, what is the chance that you will register to vote and vote for president in November 2004. Use a scale of 0 to 100. If you are certain you will register and you will vote, state "100." If you are certain you will not register, or you will register and not vote, state "0". is a 40 in 100 chance you will both register and vote, state 40, and so on.

2. Regardless of whether or not you are likely to vote in the presidential election, given what is likely to happen during the course of the campaign, on a scale of 0 to 100 what is the likelihood that you would vote for John Kerry, George Bush, or some other candidate for president?

The sum of your answers should be 100. For instance, if there is a $40 \%$ chance you would vote for John Kerry and a $40 \%$ chance you would vote for George Bush, and a $20 \%$ chance you would vote for someone else, your response should be:

$\begin{array}{cc}\text { John Kerry } & 40 \\ \text { George Bush } & 40 \\ \text { Other Candidate } & 20\end{array}$

If you are certain that you would vote for Ralph Nader (or a candidate other than Bush or Kerry), your response should be:

$\begin{array}{cc}\text { John Kerry } & 0 \\ \text { George Bush } & 0 \\ \text { Other Candidate } & 100\end{array}$

For the other demographically balanced half-sample, the two questions are designed to mimic typical poll practice.

1. Are you registered to vote?

If yes:

- Are you likely to cast a vote for a presidential candidate in the 2004 election? 
If no,

- Are you likely to register in time for the election and cast a vote for a presidential candidate in the 2004 election?

2. Regardless of whether or not you are likely to vote in the presidential election, and given what is likely to happen during the course of the campaign, for whom would you vote:

- Vote for John Kerry, the Democratic nominee for president.

- Vote for George Bush, the Republican nominee for president.

- Vote for another candidate. 